\title{
DACH1 stimulates shear stress-guided endothelial cell migration and coronary artery growth through the CXCL12- CXCR4 signaling axis
}

\author{
Andrew H. Chang, ${ }^{1,2}$ Brian C. Raftrey, ${ }^{2}$ Gaetano D'Amato, ${ }^{2}$ Vinay N. Surya, ${ }^{3}$ Aruna Poduri, ${ }^{2}$ \\ Heidi I. Chen, ${ }^{1,2}$ Andrew B. Goldstone, ${ }^{4,5}$ Joseph Woo, ${ }^{4}$ Gerald G. Fuller, ${ }^{3}$ Alexander R. Dunn, ${ }^{3}$ \\ and Kristy Red-Horse ${ }^{2}$ \\ ${ }^{1}$ Department of Developmental Biology, Stanford University School of Medicine, Stanford, California 94305, USA; ${ }^{2}$ Department \\ of Biology, Stanford University, Stanford, California 94305, USA; ${ }^{3}$ Department of Chemical Engineering, Stanford University, \\ Stanford, California 94305, USA; ${ }^{4}$ Department of Cardiothoracic Surgery, Stanford University School of Medicine, Stanford, \\ California 94305, USA; ${ }^{5}$ Department of Health Research and Policy-Epidemiology, Stanford University School of Medicine, \\ Stanford, California 94305, USA
}

Sufficient blood flow to tissues relies on arterial blood vessels, but the mechanisms regulating their development are poorly understood. Many arteries, including coronary arteries of the heart, form through remodeling of an immature vascular plexus in a process triggered and shaped by blood flow. However, little is known about how cues from fluid shear stress are translated into responses that pattern artery development. Here, we show that mice lacking endothelial Dach1 had small coronary arteries, decreased endothelial cell polarization, and reduced expression of the chemokine Cxcl12. Under shear stress in culture, Dach1 overexpression stimulated endothelial cell polarization and migration against flow, which was reversed upon CXCL12/CXCR4 inhibition. In vivo, DACH1 was expressed during early arteriogenesis but was down in mature arteries. Mature artery-type shear stress (high, uniform laminar) specifically down-regulated DACH1, while the remodeling artery-type flow (low, variable) maintained DACH1 expression. Together, our data support a model in which DACH1 stimulates coronary artery growth by activating Cxc112 expression and endothelial cell migration against blood flow into developing arteries. This activity is suppressed once arteries reach a mature morphology and acquire high, laminar flow that down-regulates DACH1. Thus, we identified a mechanism by which blood flow quality balances artery growth and maturation.

[Keywords: arteriogenesis; cell migration; coronary artery development; endothelial cell biology; mechanotransduction; vascular biology]

Supplemental material is available for this article.

Received May 5, 2017; revised version accepted June 30, 2017.

Physical forces imparted by blood flow drive artery development and remodeling, but little is known about the cellular and molecular pathways linking them during embryonic development and regeneration (Hahn and Schwartz 2009; Culver and Dickinson 2010; Dolan et al. 2012; Simons and Eichmann 2015). Arteriogenesis during embryonic development occurs when a plexus of small, highly branched vessels remodels into a linear, largebore artery. Studies of the avian and mammalian yolk sac have shown that blood flow is required for plexus remodeling (Lucitti et al. 2007). Arterial remodeling in the heart's coronary plexus directly follows initiation of blood flow and does not occur in mutants where the coronary ar-

Corresponding author: kredhors@stanford.edu Article published online ahead of print. Article and publication date are online at http://www.genesdev.org/cgi/doi/10.1101/gad.301549.117. tery does not connect to its blood flow source, the aorta (Chen et al. 2014a; Ivins et al. 2015; Volz et al. 2015). Live imaging of the yolk sac and regenerating zebrafish fins revealed that arterial remodeling involves vessel fusion and endothelial migration against blood flow, toward the growing artery (Sato et al. 2010; Udan et al. 2013; Xu et al. 2014). Furthermore, studies of cell polarization in the retina indicate that endothelial cells polarize against blood flow in regions where there is a gradient from lower to higher shear stress. This activity results in the regression of vessels experiencing the lowest flow and allocation

(C) 2017 Chang et al. This article is distributed exclusively by Cold Spring Harbor Laboratory Press for the first six months after the full-issue publication date (see http://genesdev.cshlp.org/site/misc/terms.xhtml). After six months, it is available under a Creative Commons License (Attribution-NonCommercial 4.0 International), as described at http:// creativecommons.org/licenses/by-nc/4.0/. 
of their endothelial cells to larger vessels (Franco et al. $2015,2016)$. Thus, the ability of endothelial cells to recognize flow direction and strength in a manner that influences migratory behavior is emerging as an important mechanism patterning vascular development.

Signaling molecules such as the chemokine CXCL12 and its corresponding G-protein-coupled receptor, CXCR4, direct endothelial cell migration and motility. Originally, chemokines such as CXCL12 were found to be key regulators of leukocyte trafficking, but knockout analysis revealed that its receptor, CXCR4, was important for vascular development (Tachibana et al. 1998). Subsequently, this signaling axis has been shown to play a role in multiple vascular beds through stimulating endothelial cell migration during angiogenesis and artery remodeling (Ara et al. 2005; Takabatake et al. 2009; Strasser et al. 2010; Bussmann et al. 2011; Mahadevan et al. 2014; $\mathrm{Xu}$ et al. 2014). This activity also functions during coronary vessel development. CXCL12 induces coronary endothelial cell migration onto the heart in zebrafish (Harrison et al. 2015). In mice, it was shown to be expressed around the aorta, where it is important for mediating anastomosis of plexus endothelial cells with the aortic lumen (Cavallero et al. 2015; Ivins et al. 2015). These reports also found $C x c 112$ and Cxcr4 in large intramyocardial blood vessels; however, a potential role of coronary artery-derived CXCL12 was not explored. CXCR4 has been reported to be regulated by flow (Corti et al. 2011). Thus, this signaling axis could be a candidate for regulating shear stress cell-induced endothelial cell migration during coronary artery remodeling in mammalian hearts.

In this study, we found that the transcription factor DACH1 determines artery size and is modulated by specific shear stress profiles. DACH1 is a helix-turn-helix transcription factor related to the Sno/Ski family of corepressors and was initially discovered for its role in cell specification and organ size control in Drosophila (Mardon et al. 1994). In mammals, it plays a role in determining pancreas size by supporting progenitor cell proliferation through the suppression of the cyclin-dependent kinase inhibitor P27KIP1 (Kalousova et al. 2010). DACH1 also inhibits cell cycle progression in tumor cell lines, and its suppression has been associated with poor prognosis in human breast cancer (Popov et al. 2010). DACH1 is also expressed in vascular endothelium (Gay et al. 2013; Chen et al. 2014b), but a functional role in this cell type has not been described.

Our data reveal that DACH1 is required for proper artery remodeling and that its expression stimulates endothelial cell migration against the direction of flow. We found that loss or gain of Dach1 either decreases or increases Cxc112, respectively. Furthermore, inhibition of CXCR4 abrogated Dach1-stimulated endothelial migration against flow, demonstrating that $\mathrm{Cxcl12}$ is a downstream effector. DACH1 is decreased in mature arteries that have acquired high, uniform laminar flow rates, and this type of shear stress specifically suppresses mRNA and protein levels. While it is selectively down-regulated by high laminar shear stress, regions of the vasculature experiencing low and/or nonuniform flow continue to express DACH1, including regions of human arteries that are susceptible to vascular disease. Thus, activation of DACH1 or its downstream pathways could be explored as an avenue to stimulate arterial regeneration or modulate vascular disease.

\section{Results}

Coronary artery development is disrupted in Dach1 mutants

We found that the transcription factor $\mathrm{DACH} 1$ is required for proper arteriogenesis. Previous studies identified high DACH1 expression in coronary endothelial cells during heart development (Gay et al. 2013; Chen et al. 2014b). To investigate whether DACH1 has an important functional role, we analyzed the coronary vasculature of mice with a global Dach1 knockout. Embryonic day 17.5 (E17.5) and postnatal day 0 (P0) hearts were immunostained to label endothelial cells of all blood vessels and the smooth muscle cells that surround mature coronary arteries. The coronary vasculature was then visualized through whole-mount confocal microscopy. This analysis revealed that mutant coronary arteries were smaller in caliber, lacked the normal hierarchical reduction in lumen size when moving from proximal to distal, and sometimes exhibited abnormal looping (Fig. 1A). In contrast, capillary vessels did not exhibit any gross structural abnormalities, and quantification of vessel parameters showed no significant differences (Fig. 1B,C). Quantification of mutant arteries detected a significant decrease in lumen diameter of both the left and right main coronary arteries (Fig. 1D), which was also significant when grouped by litter (Supplemental Fig. S1A,B). In contrast, smooth muscle cell coverage was normal (Fig. 1E). Measuring primary, secondary, and tertiary branches showed that the progressive reduction in diameter in mutant coronary arteries had a decreased slope when compared with wild type, suggesting a failure to establish the proper hierarchical structure (Fig. 1F). Finally, counting the number of arteries with persistent looping structures showed this to be a common feature of the mutant left coronary artery but not of those in wild-type animals (Fig. 1G), suggesting a defect in vessel coalescence during remodeling. RNA sequencing was performed on whole hearts from E17.5 knockout animals and wild-type littermates to identify differentially expressed genes. Among the genes that were decreased were those expressed in arterial smooth muscle, including Smooth muscle myosin heavy chain (SM-MHC/Myosin 11), Elastin, and Aggrecan (Supplemental Table S1). Since smooth muscle coverage was not significantly decreased (Fig. 1E), this result is likely due to the existence of smaller coronary arteries, which would contain fewer total smooth muscle cells per heart. The artery defects did not appear to be secondary to gross cardiac defects, as sections through the heart showed apparently normal structures (Fig. $1 \mathrm{H}$ ). These data reveal that DACH1 is required for coronary arteries to reach proper size and shape. 


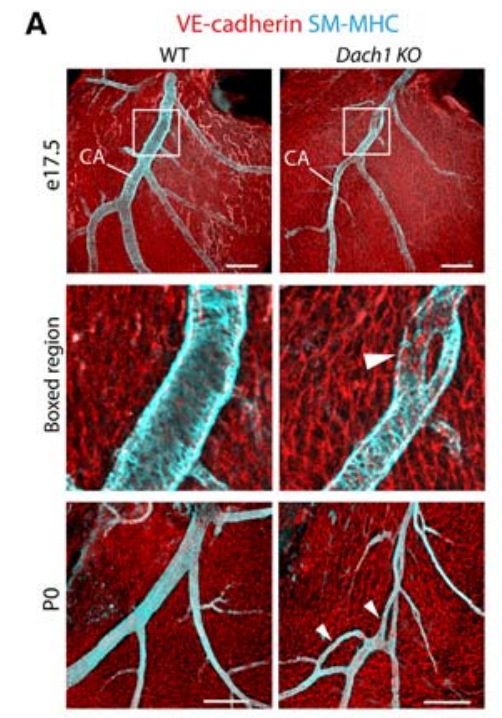

F Hierarchical patterning was defective in Dach1 KO coronary arteries (e17.5) LCA

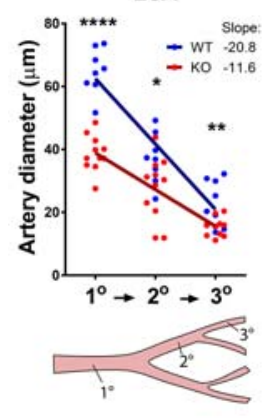

B

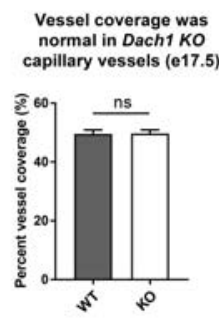

D
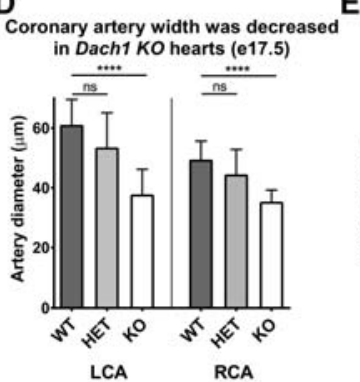

G

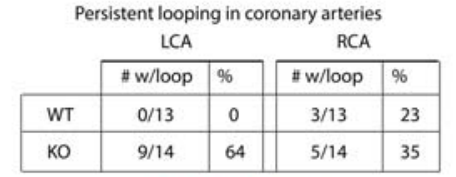

H

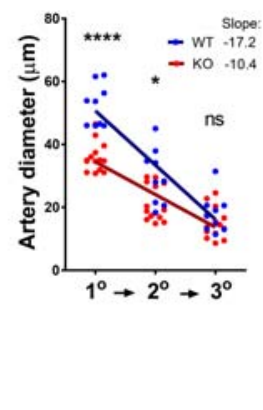

C

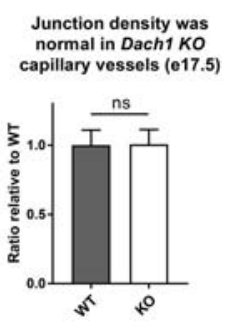

E

SMC coverage was normal

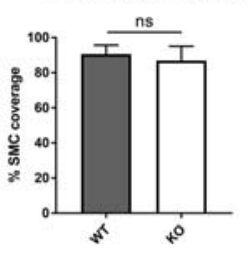

Figure 1. Dach1 mutants have small coronary arteries. $(A)$ Whole-mount confocal images of hearts from E17.5 or P0 immunolabeled with VE-cadherin (endothelial cells; red) and SM-MHC (smooth muscle; blue). Dach1 mutant coronary arteries (CA) were smaller in diameter and exhibited abnormal looping structures (arrowheads). $(B, C)$ There were no apparent structural defects in Dach1 mutant capillaries, as quantified by vessel coverage $(B)$ and junction density (C). $n=9$ wild type; $n=14$ knockout. (D) Quantification of the diameters of the left (LCA) and right (RCA) main coronary arteries (primary branches). $n=9$ wild type; $n=$ 28 heterozygous; $n=14$ knockout. $(E)$ Smooth muscle coverage of the coronary artery was unaffected at E17.5 by Dach1 deficiency. $n=8$ wild type; $n=10$ knockout. $(F)$ Linear regression lines of artery diameters when moving from primary $\left(1^{\circ}\right)$ to secondary $\left(2^{\circ}\right)$ to tertiary $\left(3^{\circ}\right)$ branches showed a shallower slope, indicating a potential defect in hierarchical patterning in knockout hearts at E17.5. Dots represent individual arteries. LCA: $n=9$ wild type, $n=11$ knockout; RCA: $n=9$ wild type, $n=4$ knockout. $(G)$ Quantification of persistent arterial loops (indicated by arrowheads in $A)$ in E17.5 hearts. (H) Representative images of tissue sections from Dach1 knockout and wild-type littermate hearts show that Dach1-deficient hearts did not display any gross structural defects. Error bars indicate standard deviation. (ns) Nonsignificant; (*) $P<0.05$; $\left.{ }^{* *}\right) P<0.01$; $\left(^{* * *}\right) P<0.0001$. Bars: $A, 200 \mu \mathrm{m} ; H, 500 \mu \mathrm{m}$.

\section{DACH1 is dynamically expressed in coronary endothelial cells during arteriogenesis}

We next investigated the expression of DACH1 during arteriogenesis. Coronary arteriogenesis begins around E13.5, once the immature coronary plexus (a web of similarly sized capillaries) attaches to the aorta, initiating blood flow (Chen et al. 2014a; Volz et al. 2015). Following this, the vessels directly downstream from the aortic attachment site begin arterial remodeling, where they coalesce and increase in size, transforming themselves over the next $2 \mathrm{~d}$ into a larger tube with fewer side branches (Fig. 2A; Volz et al. 2015). Immunofluorescence for DACH1 followed by whole-mount confocal microscopy showed that, within the heart ventricle, DACH1 was expressed exclusively in endothelial cells that line coronary vessels (Fig. 2B). It was not uniformly expressed in coronary endothelial cells. At E14.5, DACH1 was easily detected in the endothelial nuclei of vessels undergoing arterial remodeling (Fig. 2B), but quantification revealed levels to be lower than the nonremodeling capillary vessels (Fig. $\left.2 \mathrm{~B}^{\prime}\right)$. This pattern became more prominent a day later, at E15.5, when DACH1 staining was significantly decreased in the larger arteries that had emerged (Fig. 2C, $C^{\prime}$ ). DACH1 down-regulation was even more extensive at E16.5, when more arteries had completed the remodeling phase (Fig. $\left.2 \mathrm{D}, \mathrm{D}^{\prime}\right)$. This pattern of expression (high in smaller vessels and low in large arteries) persisted in adult hearts (Fig. 2E) and was also detected in retinal arteries (Supplemental Fig. S2). These expression patterns indicate that DACH1 likely functions within coronary endothelial cells, but its activities are down-regulated as arteries become mature.

Since DACH1 levels were decreased in arteries and these were the only vessels obviously impacted in mutant hearts, we investigated possible mechanisms of arterial growth. Endothelial proliferation rates at E14.5 were assessed using 5-ethynyl-2'-deoxyuridine (EdU) incorporation assays, which identified the number of cells in $S$ phase at a given time point. EdU incorporation was highest in the subepicardial plexus, located on the surface of the heart, and decreased in the plexus endothelial cells deeper within ventricular heart muscle (intramyocardial layer). EdU incorporation decreased even further in regions of the intramyocardial plexus that had initiated arterial remodeling, and vessels that had achieved the mature artery shape were devoid of proliferating cells (Fig. 2F,G). Low proliferation rates in arterial vessels during developmental time points when the vessels are still 
A
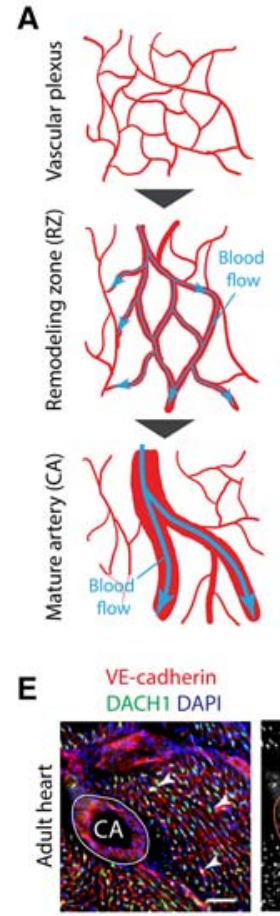

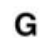

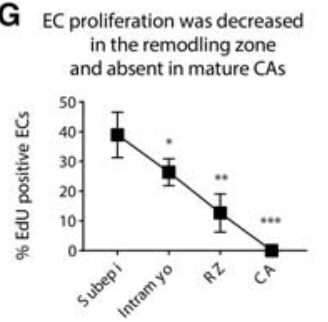

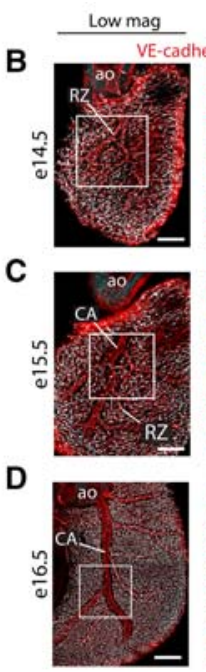
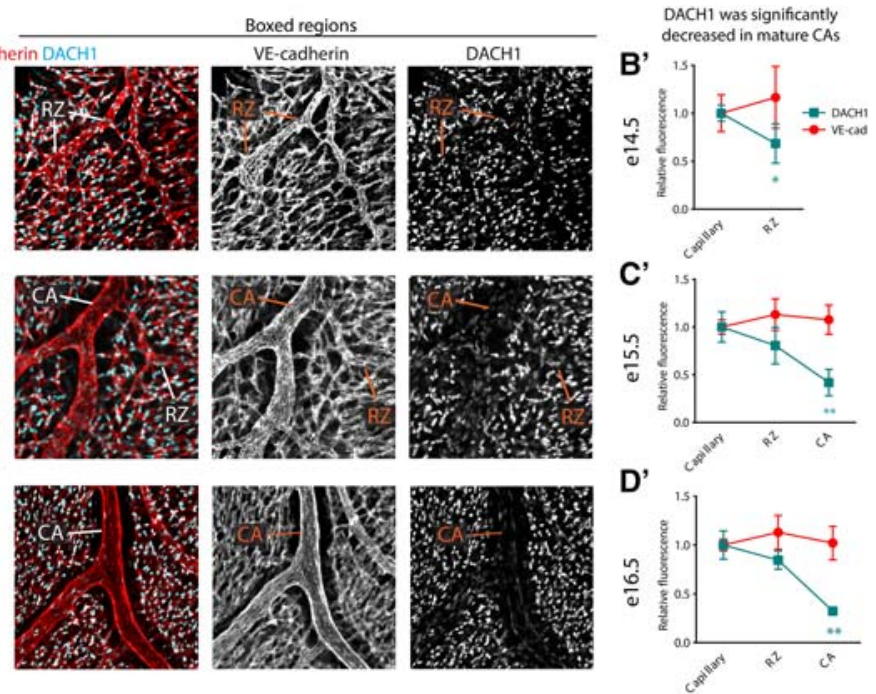

Vessel location

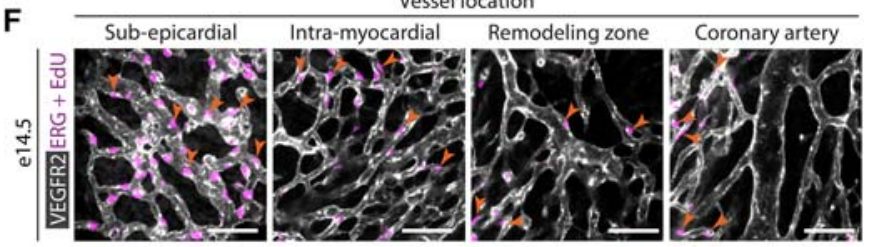

H

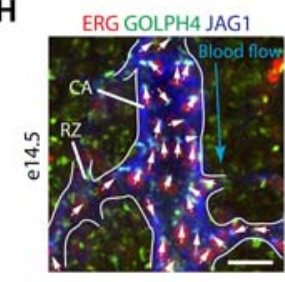

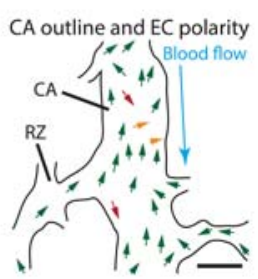

I

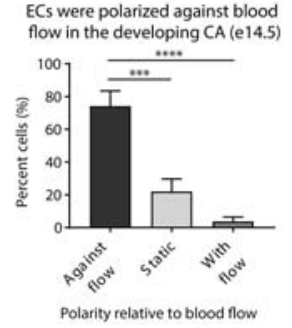

Figure 2. DACH1 is expressed in coronary endothelial cells. (A) Schematic of arterial remodeling where small plexus vessels create a remodeling zone (RZ) that transforms into a mature coronary artery (CA). Blue arrows indicate relative direction and magnitude of blood flow. $(B, C, D)$ Whole-mount confocal images localizing DACH1 during arteriogenesis of the right coronary artery at developmental stages E14.5 (B), E15.5 $(C)$, and E16.5 (D). Boxed regions (middle) highlight remodeling zones undergoing arteriogenesis in the low-magnification views (left). DACH1 was localized to coronary endothelial cells but decreased in arteries as they matured, while VE-cadherin levels were unchanged. $\left(B^{\prime}, C^{\prime}, D^{\prime}\right)$ Quantification of fluorescent intensity. $n=6$ hearts per time point. (E) Tissue section from an adult heart showing the absence of DACH1 in endothelial cells of large coronary arteries but maintenance of expression in capillaries (arrowheads). (F) Representative images of proliferating endothelial cells (arrowheads) in different regions of the coronary vasculature. Colocalization analysis was used to exclude 5-ethynyl-2'-deoxyuridine-positive $\left(\mathrm{EdU}^{+}\right)$staining in nonendothelial cells (see the Materials and Methods). (G) Quantification of endothelial proliferation rates shown in $F . n=4$ wild-type hearts. Statistical comparisons are between the subepicardial and the indicated regions. (H) Endothelial polarity in developing vessels was determined by the orientation of the nuclei (ERG) and Golgi (GOLPH4) relative to the direction of local blood flow from the aorta. Arrows indicate either polarization against flow (green), static/unpolarized (yellow), or polarization with flow (red). (I) Quantification of polarity as indicated in $H . n=4$ wild-type arteries. (Ao) Aorta; (EC) endothelial cell. Error bars indicate standard deviation. $\left.\left.\left.\left(^{*}\right) P<0.05 ;{ }^{* *}\right) P<0.01 ;{ }^{* * *}\right) P<0.001 ;{ }^{* * * *}\right) P<0.0001$. Bars: $B, C, 200 \mu$ m; $D, 300$ $\mu \mathrm{m} ; E, 100 \mu \mathrm{m} ; F, 50 \mu \mathrm{m} ; H, 25 \mu \mathrm{m}$.

expanding have been observed in other systems (Ehling et al. 2013; Udan et al. 2013). These observations suggest that the expansion of developing arteries is thus unlikely due to the proliferation of endothelial cells already in the maturing vessel.

Analyses in the yolk sac and retina have shown that arterial remodeling is coincident with endothelial polarization and migration against blood flow (Udan et al. 2013; Franco et al. 2015, 2016), suggesting that, instead of in situ proliferation, arterial expansion proceeds through the recruitment of cells from the surrounding capillaries. We next aimed to gain evidence that coronary artery growth uses a similar mechanism. Coronary arteries cannot be visualized using time-lapse microscopy. Therefore, endothelial cell polarity was used as an indicator of the direction of cell migration. E14.5 hearts were immunolabeled for JAG1 to outline remodeling arteries, ERG to label endothelial nuclei, and GOLPH4 to mark polarity. 
Golgi location relative to the nucleus was then used to distinguish polarity (Fig. 2H; Franco et al. 2015, 2016; Wang et al. 2016). We found that the majority of endothelial cells within the remodeling artery was polarized against the direction of blood flow (Fig. 2H,I). These cells could be either migrating against flow or nonmigratory with directional polarization. However, the pattern of proliferation and polarity data together support a model in which arteries expand through migration and/or incorporation of surrounding plexus endothelial cells. This suggests that the small artery phenotype in Dach1 mutants could arise from a migration defect in plexus and remodeling endothelial cells, where it is highly expressed in wild-type hearts.

\section{DACH1 functions in endothelial cells to affect cell polarity during artery remodeling}

Experiments were next performed to confirm whether DACH1 functions in endothelial cells to affect artery size. A Dach1 conditional knockout line (Dach $\left.1^{\text {flox/flox }}\right)$ was obtained (see the Materials and Methods) and crossed with the endothelial-specific Tie2Cre deletion line, creating Tie2Cre; Dach $1^{\text {flox/flox }}$ mice. This endothelial conditional knockout is referred to here as Dach1 eCKO. Immunostaining showed that this line effectively and specifically abolished DACH1 expression in the coronary endothelial cells in the Dach1 eCKO animals compared with their Cre-negative control littermates (Fig. 3A). Furthermore, the specific loss of endothelial DACH1 recapitulated the small coronary artery phenotype from the Dach1 global knockout (cf. Figs. 3B, and 1A). Quantification of the arterial diameter showed that the Dach1 eCKO animals had significantly smaller caliber when statistical comparisons were made by either grouping all animals or grouping within litters (Fig. 3C; Supplemental Fig. S3A,B). As in global Dach1 knockouts, smooth muscle coverage was not affected at this stage (Fig. 3D). In contrast to the Dach1 knockout animals, Dach1 eCKOs survived into adulthood at the expected ratios $(n=80)$ but with compromised heart function (Supplemental Fig. S3C). Thus, $\mathrm{DACH} 1$ is required in endothelial cells to obtain proper artery size and support adult heart function.

Because the primary defect in Dach1-deficient animals was small coronary arteries, we analyzed mutant hearts throughout embryonic development to identify when the defect arises. Formation of the early coronary plexus was not affected in mutant hearts (data not shown), and the capillary vessels were not affected throughout development (Supplemental Fig. S3D,E). In contrast, there were differences detected in vessels undergoing artery remodeling. Whole-mount immunostaining for all endothelial cells (VE-cadherin) and those undergoing remodeling (JAG1) (Volz et al. 2015) made it possible to categorize early remodeling zones, late remodeling zones, and mature arteries (Fig. 3E). Classifying immunolabeled hearts from stages E13.5 to E15.5 showed that maturation of the remodeling zone was delayed in Dach1 knockout hearts (Fig. 3F). Arteries in Dach1 eCKO animals were also delayed at E14.5 (Fig. 3F, rightmost columns). In contrast to the normal smooth muscle coverage at E17.5, there was a decrease at E15.5, likely due to the remodeling delay (Supplemental Fig. S3F). By E17.5, all arteries were mature, showing that remodeling was complete in mutant hearts (Fig. 3F, E17.5 time point) but that their final sizes were significantly smaller (see Fig. 1D). The delay in remodeling did not result from a delayed onset of blood flow, since connections to the aorta (Supplemental Fig. S3G) and expression of the flow-responsive ligand JAG1 (Volz et al. 2015) were similar in controls and knockouts (Fig. 3E, cf. knockout vs. wild type). These data show that DACH1 functions in endothelial cells to allow robust progression of arterial remodeling.

Given the expression pattern of DACH1 and the mechanism of artery growth (see Fig. 2), we hypothesized that the remodeling delay could be due to a decreased ability of Dach1-deficient endothelial cells to migrate or incorporate into the developing artery, thus stunting its growth. Cell polarity was therefore analyzed at the coronary artery remodeling zone as an indicator of the cells' potential migratory direction (Fig. 3G). Compiling cell polarity measurements from the entire $\mathrm{JAG}^{+}$artery in each heart showed that Dach1 eCKO animals had a significantly lower percentage of cells polarized against blood flow (Fig. 3H). There was also a corresponding increase in the fraction of cells in the static/unpolarized state (Fig. $3 \mathrm{H})$. Similar results were obtained in global Dach1 knockout animals (data not shown). Defects in polarization could result in inefficient directed migration. To directly test a role for DACH1 during cell migration, lentiviral delivery of Dach1-specific shRNAs was used to decrease mRNA and protein expression in primary human coronary artery endothelial cells (HCAECs) (Supplemental Fig. S3H,I). This manipulation caused a decrease in directed migration in transwell migration assays (Fig. 3I). These data support a potential role for $\mathrm{DACH} 1$ in stimulating cell polarity and migration against blood flow into the remodeling artery, effectively stimulating artery growth.

\section{Ectopic expression of Dach1 stimulates shear stress-guided cell behaviors}

To further explore the role of DACH1 in endothelial cells, we next overexpressed Dach1 and analyzed its effects on various aspects of endothelial cell behavior. A lentiviral vector was used to produce endothelial cells that express either GFP or Dach1-GFP under the control of a constitutive promoter ( $\beta$-actin) (Supplemental Fig. S4A). Because $\mathrm{DACH} 1$ is a transcription factor, we first explored the genes that are regulated by it. mRNA was isolated from endothelial cells (HCAECs) transduced with either Lenti-Dach1-GFP or Lenti-GFP and subjected to RNA sequencing. Dach1 overexpression induced 438 genes and decreased 343 genes when a false discovery rate (FDR)-adjusted $P$-value of 0.001 was used as a cutoff (Supplemental Table S2). A subset of these genes was validated using quantitative PCR (qPCR) (Supplemental Fig. S4B). Analysis of the top 20 biological process gene ontology (GO) terms revealed that $\mathrm{DACH} 1$ regulates genes involved in cell migration/motility, cell-cell signaling, and vascular development (Fig. 4A). Three of the top seven GO terms 
A
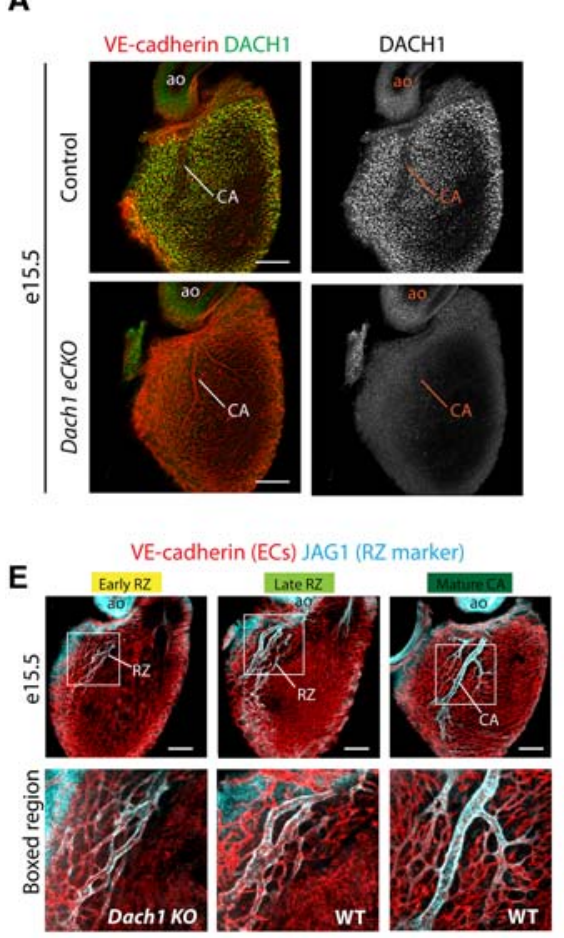

$\mathbf{F}$

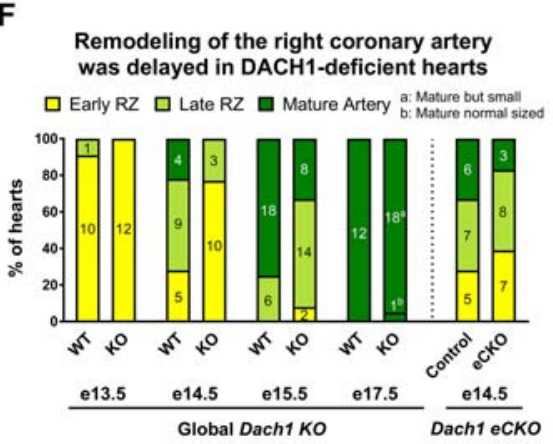

B

B

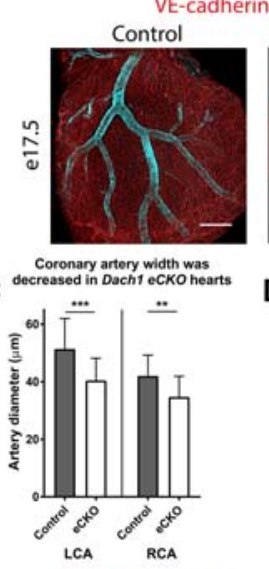

G

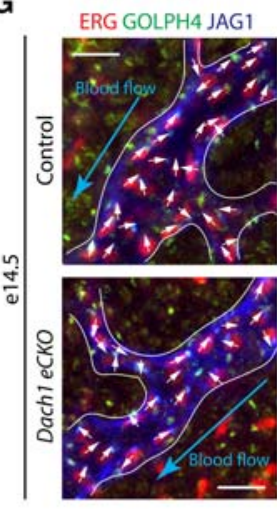

H Cell polarity was decreased in the hodeling coronary arteries from

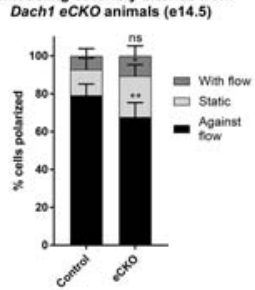

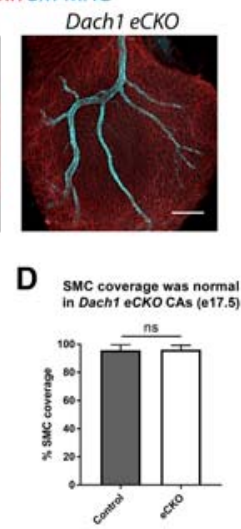

CA outline and EC polarity
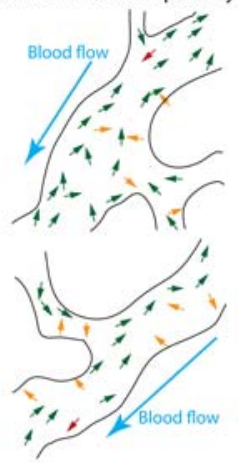

I Endothelial colls depleted tor Dach1 exhibited
impaired migration

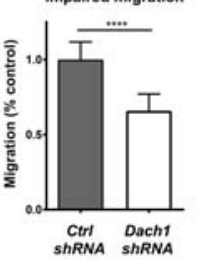

Figure 3. Endothelial-specific deletion of Dach1 disrupts arterial remodeling and endothelial cell polarization. (A) DACH1 was completely eliminated in the endothelial cells of Dach1 eCKO mice. (B) Dach1 eCKO coronary arteries recapitulated the smaller arterial diameter phenotype present in those of global Dach1 knockouts. (C) Quantification of the diameters of the left (LCA) and right (RCA) main coronary arteries at E17.5. $n$ $=32$ control; $n=18$ Dach1 eCKO. (D) Smooth muscle coverage of the coronary artery was unaffected by endothelial Dach1 deficiency at E17.5. $n=11$ control; $n=7$ Dach1 eCKO. (E) Classification of $\mathrm{JAG}^{+}$arterial remodeling zones (early, late, and mature) in whole-mount confocal images at E15.5 during coronary arteriogenesis. Genotypes of hearts are indicated. $(F)$ Classifying remodeling zones in wildtype and Dach1 mutant hearts revealed a delay in remodeling that was resolved by E17.5 but resulted in smaller arteries. A remodeling delay was also present in the Dach1 eCKOs at E14.5. The number of hearts classified is shown. $(G)$ Representative images of endothelial polarity quantification in mutant coronary arteries. Arrows indicate against flow (green), unpolarized/static (yellow), or with flow (red). (H) Quantification of polarity as indicated in $G$ showed a reduction in polarity against flow in Dach1 eCKO arteries. $n=7$ control arteries; $n=9$ Dach1 eCKO arteries. Statistical comparisons are between the same categories in control and Dach1 eCKO. (I) Migration in human coronary artery endothelial cells (HCAECs) was decreased when Dach1 was depleted using lentiviral-delivered Dach1-specific shRNAs. (Ao) Aorta; (CA) coronary artery; (EC) endothelial cell; (RZ) remodeling zone. Error bars indicate standard deviation. (ns) Nonsignificant; $\left(^{*}\right) P<0.05 ;\left({ }^{* *}\right) P<0.01 ;\left({ }^{* *}\right) P<0.001 ;\left({ }^{* * * *}\right) P<0.0001$. Bars: $A$, $B, E, 200 \mu \mathrm{m} ; G, 25 \mu \mathrm{m}$.

implicated DACH1's role in cell movement, further suggesting a role in cell migration at the arterial remodeling zone.

Since the endothelial cells at the remodeling zone are heavily influenced by the mechanical forces of blood flow, we next aimed to assess the response of Dach1overexpressing cells to experimental fluid shear stress. HCAECs were exposed to uniform laminar shear stress using a parallel plate flow chamber attached to a fluidic unit/pump system that continuously flows medium parallel to the culture surface at 35 dynes $/ \mathrm{cm}^{2}$. Tracking the migration of individual cells over the 24- to $72-\mathrm{h}$ time period showed that Dach1-overexpressing cells migrated against flow to a much greater extent compared with controls (Fig. 4B; Supplemental Videos 1, 2). Averaging total migration along the axis relative to flow direction ( $Y$-axis) showed a significant increase in migration against flow in the presence of Dach1 overexpression (Fig. 4C). The effect of Dach1 was specific for directional migration, since total migration and velocity were not different among the two cell populations (Fig. 4C; Supplemental Fig. S4C). Cells behaved similarly at other shear stress magnitudes, including 20 dynes $/ \mathrm{cm}^{2}$ (data not shown). These data indicate that Dach1 stimulates directional cell migration in response to shear stress-induced signals.

DACH1 also influenced other shear stress-induced cell behaviors in addition to stimulating migration against flow. Analysis of time-lapse images indicated that Dach1 overexpression potentiated endothelial cell alignment (Fig. 4D). Measuring the angle between the long axis of the cell and the direction of flow at different time points revealed that Lenti-Dach1 HCAECs were more aligned than control cells and began aligning earlier following the onset of flow (Fig. 4E). In addition, the Dach1- 
expressing cells were also more elongated and began lengthening along the axis of flow at an earlier time in culture (Fig. 4F). Cell polarity was also assessed at the 72-h endpoint by measuring the Golgi position relative to the nuclei and sorting the cells into an "against flow," "static," or "with flow" category based on their angle relative to flow direction (Fig. 4G,H). This analysis revealed that there was a higher percentage of cells polarized against flow under Dach1 overexpression conditions (Fig. 4I). There was also an increased percentage of highly polarized cells; i.e., within $15^{\circ}$ of the flow line (Fig. 4J). Taken together, the cell culture experiments showed that Dach1 expression stimulated migration, polarization, and alignment against blood flow, which are behaviors that would support artery growth in vivo (Sato et al. 2010; Udan et al. 2013).

The CXCL12/CXCR4 signaling axis acts as a downstream effector of DACH1

Next, we sought to find the downstream molecules propagating DACH1's promigratory and polarizing effects on cells in order to elucidate the mechanism stimulating arteriogenesis during remodeling. One of the most significantly changed genes in global Dach1 knockout hearts according to our RNA sequencing data was Cxcl12 (Fig. 5A; Supplemental Table S1). Conversely, Cxc112 was upregulated in HCAECs overexpressing Dach1 as assessed by RNA sequencing and qPCR (Fig. 5B; Supplemental Table S2; Supplemental Fig. S4B). To determine whether Cxcl12 is down-regulated in endothelial cells of Dach1 knockout hearts, Dach1 knockout mice were bred to a Cxcl12-DsRed reporter line. This readout showed that while wild-type hearts have high levels of Cxcl12 in both the coronary artery and adjacent remodeling zones, Dach1 knockout littermates have significantly less expression at the same regions (Fig. 5C,D). Furthermore, at late gestation and postnatal time points, Cxcl12-Dsred was down-regulated specifically in large arteries where DACH1 was reduced (data not shown). In situ hybridization for Cxcl12 in Dach1 eCKO animals also showed decreased endothelial expression (Fig. 5E). These data show that Dach1 is upstream of endothelial Cxcl12 expression in vitro and in vivo.

Experiments were next performed to investigate whether the CXCL12-CXCR4 signaling axis regulates the Dach1-driven cell behaviors described in Figure 4. With this goal, we performed the same parallel plate experiment with Lenti-GFP and Lenti-Dach1-overexpressing endothelial cells (Fig. 4B) with the addition of AMD3100, a specific CXCR4 blocker (Schols et al. 1997). CXCR4 inhibition attenuated migration against flow in Lenti-Dach1 cells (Fig. 5F). This was specific to Dach1 overexpression, since AMD3100 did not significantly change migration in Lenti-GFP cells (Fig. 5F). An assessment of cell polarity at the end of the culture period showed that inhibiting CXCR4 decreased the number of cells polarized against flow in Lenti-Dach1-expressing cells. However, this was not specific to Dach1-overexpressing cells, as a similar decrease was seen in controls
(Fig. 5G). This suggests that activation of CXCR4 by CXCL12 stimulates endothelial cell polarization in response to shear stress but that this activity is independent of DACH1. Thus, CXCL12 appears to be one of the key components downstream from DACH1 that stimulates cell migration against the direction of flow.

\section{DACH1 is down-regulated by arterial-type shear stress in vitro}

Another notable aspect of DACH1 that we sought to understand is the mechanism behind DACH1's change in expression from high at the capillary plexus to low in the mature coronary artery (Fig. 2B-D). One possible mechanism for the differential expression patterns is the variation in blood flow profiles found in these different regions (Hahn and Schwartz 2009). For example, while large arteries generally provide a smooth path, allowing for high, uniform laminar shear stress, remodeling vessels are highly branched, resulting in lower, nonuniform shear stress. We used the fluidic unit and parallel plate chamber system described in Figure 4 to mimic the flow found in large arteries (Fig. 6A). Cells cultured under flows that induced shear stress between 5 and 35 dynes $/ \mathrm{cm}^{2}$ stimulated a robust, dose-responsive down-regulation of DACH1 when compared with static controls (Fig. 6B). DACH1 levels declined by $60 \% \pm 9 \%$ at the highest flow rates tested (Fig. 6C), which was similar to the $68 \% \pm$ $4 \%$ decrease seen when comparing capillaries with mature arteries in vivo (Fig. 2D'). Similar results were obtained under either continuous or pulsatile uniform laminar flow (data not shown). Importantly, changes in DACH1 levels were not due to differences in cell density, a parameter that can affect genes sensitive to mechanotransductive forces (Supplemental Fig. S5A). Orbital rotation was carried out as a second method of imparting high laminar shear stress on endothelial cells (Dardik et al. 2005; Paz et al. 2012) in order to obtain sufficient quantities of material to perform mRNA and protein quantification experiments. Exposing endothelial cells to this type of shear stress for $24 \mathrm{~h}$ caused DACH1 protein to decrease by $68 \% \pm 18 \%$ (Supplemental Fig. S5B,C) and Dach1 mRNA to decrease by $53 \% \pm 14 \%$ (Supplemental Fig. S5D). These results show that high, uniform laminar shear stress elicits a strong decrease in Dach1 expression, providing a possible mechanism underlying its lower levels in mature arteries.

Since DACH1 is still high in remodeling arteries and capillaries, which are also perfused with blood, we hypothesized that the type of shear stress present in these vessels is not capable of robustly inhibiting Dach1 expression. To gain evidence for this hypothesis, endothelial cells were exposed to gradient (nonuniform) shear stress (Fig. 6D), which more closely models the forces experienced by early remodeling arteries with their tortuous vessels and branch points (Fig. 2A). Cells were cultured using an impinging flow device that jets medium perpendicular to the culture surface, creating regions where shear stress accelerates from 0 to 35 dynes $/ \mathrm{cm}^{2}$ over $\sim 10$ cell lengths before decelerating back to negligible flow 
DACH1 in shear stress responses and arteriogenesis

A

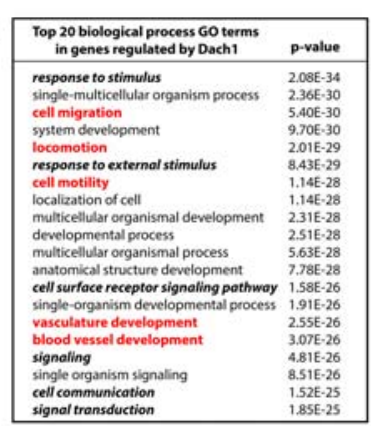

D

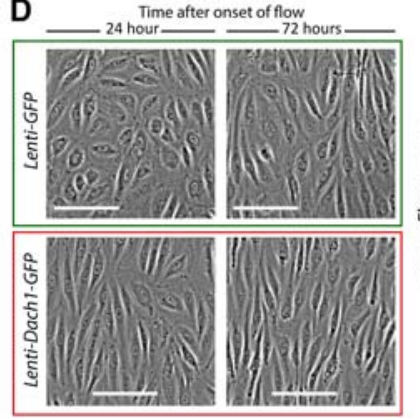

G

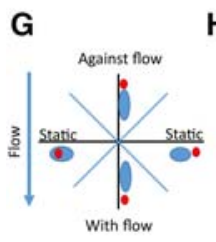

H

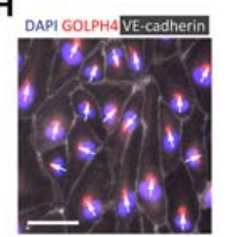

B

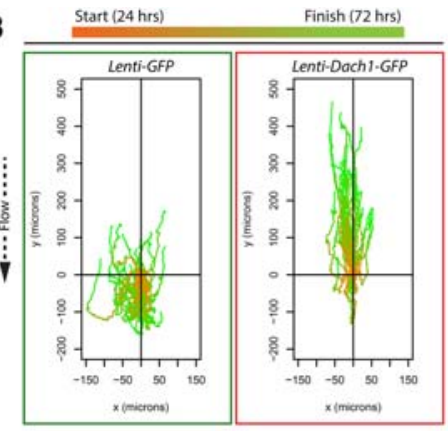

C Dach1 overexpression induced migration against flow

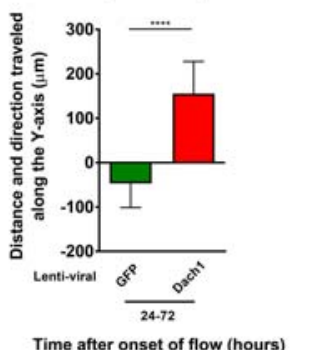

E
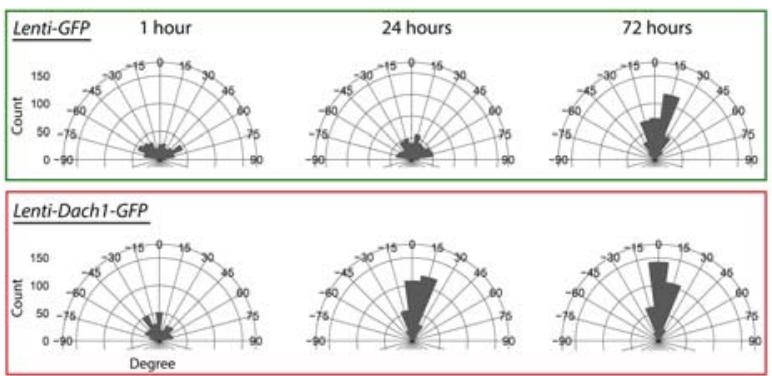

Dach1 overexpression did not alter general cell migration

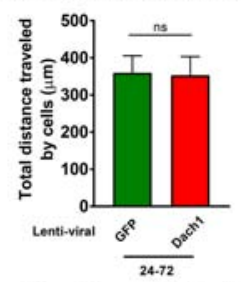

Time after onset of flow (hours)

F

Endotherial cell elongation was enhanced upon Dach1 overexpression

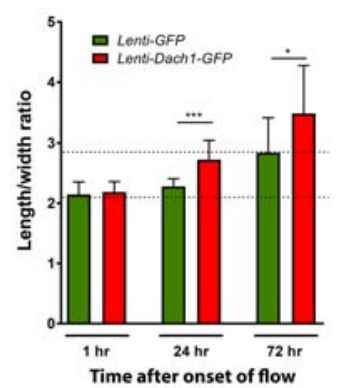

Time after onset of flow

J

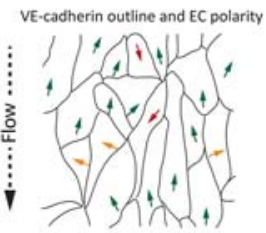

I Dach1 overexpression enhanced polarity against flow (72hr)
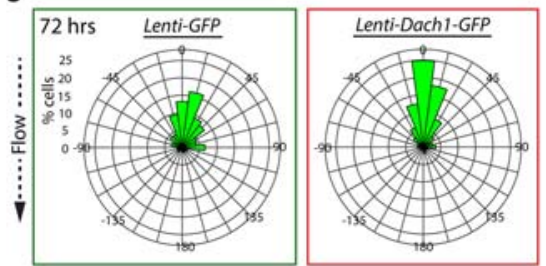

Figure 4. Dach1 overexpression stimulates shear stress-guided endothelial cell behaviors. $(A)$ List of GO terms enriched in Dach1-overexpressing cells, indicating a prominent role in cell motility (highlighted in red). Cell-cell signaling-related terms are in bold italic type. $(B-F)$ HCAECs were infected with either Lenti-GFP or Lenti-Dach1-GFP, cultured under uniform laminar shear stress $\left(35 \mathrm{dyn} / \mathrm{cm}^{2}\right)$, and subjected to time-lapse imaging. (B) Plotting migration tracks (40 cells per condition) showed that Lenti-Dach1-GFP cells migrated against flow throughout the culture period. $(C)$ Migration along the $Y$-axis and total migration. $n=120$ cells from three experiments. $(D)$ Still images from time-lapse movies showing that Dach1-GFP cells aligned earlier than controls. $(E)$ Rose plots depicting cell alignment angle at different time points following the onset of flow. $n=\sim 350$ cells from one representative experiment of three total experiments. $(F)$ Measurements of cell length versus width showing that Dach1 expression enhanced elongation in response to flow. $(G-J)$ HCAECs were infected with either Lenti-GFP or Lenti-Dach1-GFP, cultured under uniform laminar shear stress for $72 \mathrm{~h}$, and immunostained to label Golgi and nuclei. $(G)$ Schematic showing how cell polarity was quantified using localization of nuclei (blue) and Golgi (red). $(H)$ Example of categorization as described in G. Arrows indicate cells oriented against flow (green), static/unpolarized cells (yellow), or cells oriented with flow (red). (I) Lenti-Dach1-GFP cells exhibited a significantly increased percentage of cells in the "against flow" group relative to control cells. $(J)$ An increased percentage of highly polarized cells was also evident. Error bars indicate standard deviation. (ns) Nonsignificant; $\left({ }^{*}\right) P$ $<0.05 ;\left(^{* *}\right) P<0.01 ;\left(^{* * *}\right) P<0.001 ;\left(^{* * *}\right) P<0.0001$. Bars: $D, 100 \mu \mathrm{m} ; H, 50 \mu \mathrm{m}$.

rates $1.25 \mathrm{~cm}$ away from the impingement point (Fig. 6D; Ostrowski et al. 2014). Cells cultured under impinging flow only mildly decreased their DACH1 expression even at the highest shear stress magnitudes (Fig. 6E,F). DACH1 expression was similar at all gradient flow rates $>3$ dynes $/ \mathrm{cm}^{2}$, which was $80 \%$ of control regions (those with absent or negligible flow rates) (Fig. 6G). This was in contrast to uniform laminar flow, which continuously decreased DACH1 at shear stresses up to 20 dynes $/ \mathrm{cm}^{2}$ (Fig. 6G). Again, cell density could not explain differences in DACH1 in the gradient flow system, since the small changes in this parameter among regions did not match the pattern of small changes in expression levels (cf. Supplemental Fig. S5E and Fig. 6F). Together, these data indicate that DACH1 is down-regulated specifically by uniform laminar shear stress, consistent with its lower expression pattern specifically in large arteries.

\section{DACH1 is expressed at branch points in vitro and in vivo}

Arterial branch points are another blood vessel location with distinct shear stress patterns, prompting us to investigate DACH1's presence in these regions. Here, vessel divisions redirect blood flow in a manner that creates areas 

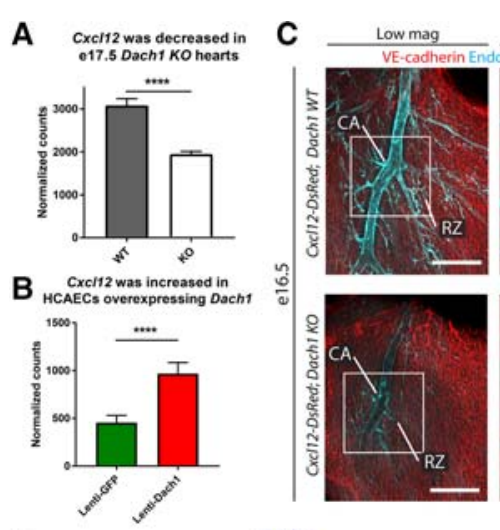

E

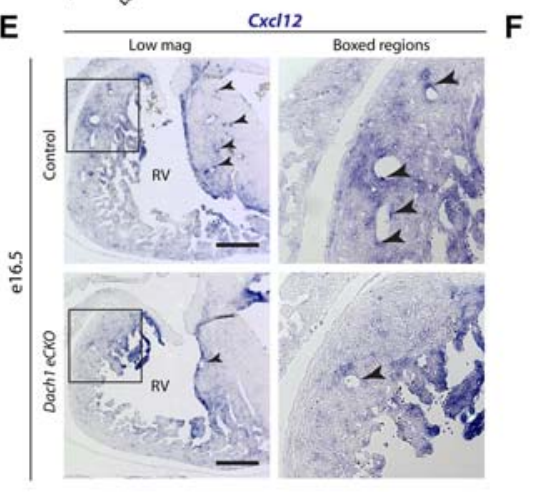

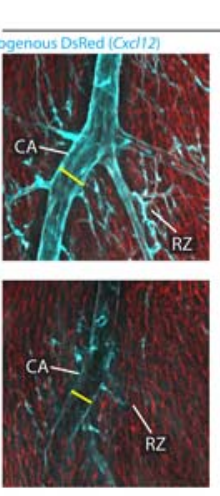

F

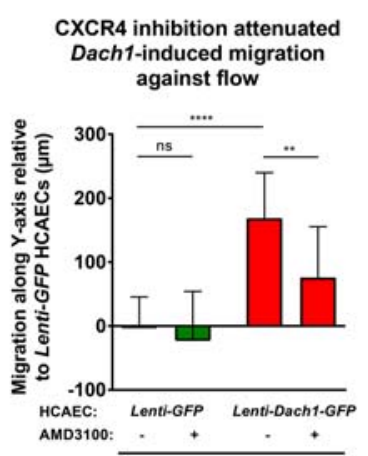

$24-72$ hours after onset of flow

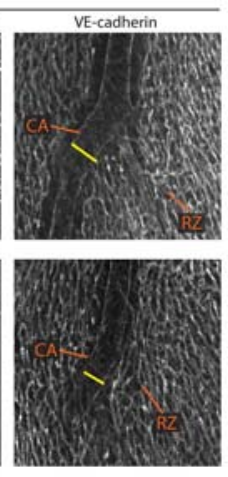

D

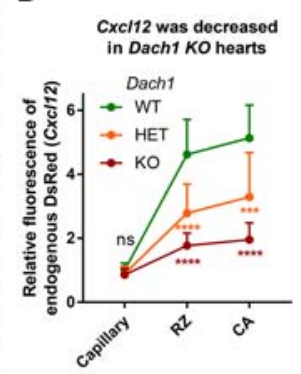

G

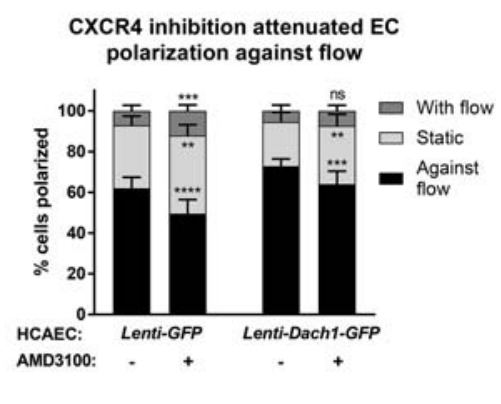

Figure 5. The CXCL12-CXCR4 signaling axis is downstream from DACH1. (A) RNA sequencing data from whole E17.5 hearts revealed that $C x c 112$ was down-regulated in Dach1 knockout animals. $n=3$ wild-type hearts; $n=3$ knockout hearts. $(B)$ RNA sequencing from HCAECs overexpressing Dach1 showed that Cxcl12 was up-regulated relative to controls. $n=3$ biological replicates per condition. (C) The Cxcl12 reporter gene (DsRed) was decreased in Dach1 knockout hearts. Artery widths are indicated by yellow lines. $(D)$ Quantification of endogenous DsRed fluorescence. $n=3$ wild type; $n=8$ heterozygous; $n=5$ knockout. $(E)$ In situ hybridization for $C x c 112$ in Dach1 $e C K O$ hearts showed decreased expression in arterial endothelial cells (arrowheads). $n=9$ control; $n=5$ Dach $1 e C K O$. (F) CXCR4 inhibition with AMD3100 reduced the migration against flow induced by Dach1 overexpression. $n=120$ cells total from three independent experiments. $(G)$ AMD3100 reduced the percentage of cells polarized against flow in all conditions. Statistical comparisons comparing the same category of cells between control and drug-treated HCAECs within the same lentiviral treatment. Error bars indicate standard deviation. (ns) Nonsignificant; $\left(^{* *}\right) P<0.01 ;\left(^{* * *}\right) P<0.001 ;\left(^{* * * *}\right) P<0.0001$. Bars, $200 \mu \mathrm{m}$.

of low and nonuniform shear stress. This phenomenon was recapitulated in culture by conducting flow experiments using a Y-shaped chamber slide that contains both straight and branching regions (Fig. 7A). We subjected HCAECs to flow at 35 dynes $/ \mathrm{cm}^{2}$ in the Y-shaped chamber for $24 \mathrm{~h}$ and compared DACH1 protein levels among the different regions. In agreement with the above experiments, straight regions experiencing uniform laminar flow had significantly lower levels of DACH1 than branching areas exposed to nonuniform laminar shear stress (Fig. 7B,C). Differences in DACH1 levels were also observed when comparing straight regions experiencing low versus high uniform shear stress. Because the branch divides fluid into two conduits equal in size to the original (thus doubling the total volume), cells at straight regions downstream from the branch experience half the levels of shear stress $\left(17.5\right.$ dynes $\left./ \mathrm{cm}^{2}\right)$ relative to those upstream of the split. These areas exhibit an intermediate level of DACH1 expression (Fig. 7C; Supplemental Fig. S6A). Endothelial cells therefore calibrate their levels of DACH1 expression in response to changes in both the uniformity and magnitude of shear stress.
To gain evidence that distinct flow types differentially regulate DACH1 expression in vivo, we investigated whether levels were changed at arterial branches, which experience low and/or nonuniform shear stress. This analysis was carried out using the developing retinal vasculature because the entire arterial network is easily visualized in whole-mount preparations. Confocal imaging of the retinal vasculature (P7 to adult) immunostained for DACH1 and VE-cadherin revealed that nuclei at branch points expressed increased levels of DACH1 (Fig. 7D; Supplemental Fig. S6B). Quantification showed that levels were significantly increased over neighboring arterial cells either upstream of or downstream from the branch point (Fig. 7E; Supplemental Fig. S6C) in 93\% of the branches analyzed. A similar pattern of DACH1 expression was found in mouse P0 coronary arteries at small branches stemming from the main vessel (Fig. 7F). These observations support the hypothesis that different types of shear stress directly affect levels of DACH1 expression in the intact vasculature.

We next tested whether DACH1 was increased at branches in human arteries. Human coronary artery 

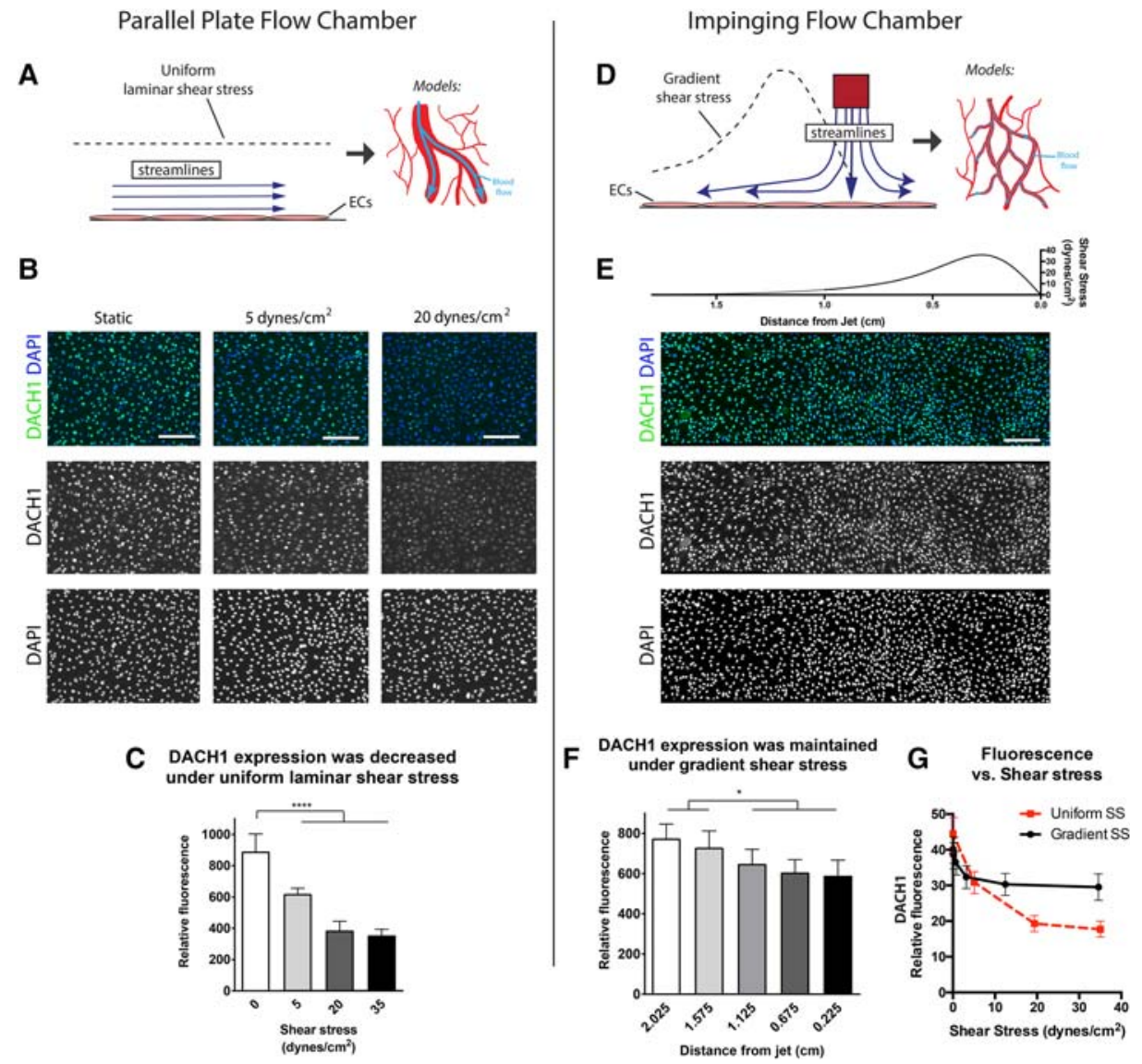

Figure 6. DACH1 is specifically decreased by arterial-type flow. (A) Schematic of a parallel plate flow chamber that models arterial-type flow (uniform laminar). (B) DACH1 immunofluorescence showed that uniform laminar flow decreased expression compared with static controls. DAPI is in blue. $(C)$ Nuclear DACH1 was significantly reduced as shear stress increased. $n=8$ or more fields of view (FOVs) per condition from three experiments. $(D)$ Schematic of impinging flow chamber that models flow in the early remodeling plexus and vascular branch points (nonuniform gradient). (E) DACH1 immunofluorescence was not obviously changed in regions experiencing gradient shear stress. $(F)$ Quantification showing that nuclear DACH1 was only mildly reduced in areas of gradient shear stress. $n=14$ FOVs per position from seven experiments. $(G)$ DACH1 levels plotted as a function of shear stress (SS) in parallel and impinging flow. Error bars indicate standard deviation. $\left(^{*}\right) P<0.05 ;\left(^{* * * *}\right) P<0.0001$. Bars, $150 \mu \mathrm{m}$.

samples were obtained, whole-mount-immunostained for $\mathrm{DACH} 1$, and imaged en face in an orientation that allowed identification of branching regions (Fig. 7G). The results showed that DACH1 was significantly increased in endothelial cells adjacent to branch openings (Fig. 7G,H; Supplemental Fig. S6D). Branch points are known to be highly susceptible to atherosclerotic lesion formation (Hahn and Schwartz 2009). Thus, these data provide additional evidence of DACH1's regulation by specific flow types and suggest a potential role in the human vascular biology.

\section{Discussion}

The mature vascular system arises through pruning and remodeling of a highly branched immature vascular plexus into hierarchically organized arteries, capillaries, and veins, all under the influence of blood flow. The regulation of these processes by blood flow has long been thought to create a vasculature optimized for efficient fluid delivery, but a detailed understanding of how mechanical signals shape vessel structure during development is lacking. Here, we present evidence that DACH1 is an endothelial cell-expressed transcription factor that supports coronary artery growth through its regulation of blood flow-guided endothelial cell behaviors. Global and endothelial-specific deletion of Dach1 results in small coronary arteries, a delay in plexus remodeling, decreased polarization of endothelial cells toward growing arteries, and lower Cxc112 expression. Under in vitro conditions mimicking blood flow-induced shear stress, Dach1-overexpressing endothelial cells become highly aligned and polarized against flow. Dach1-overexpressing cells also migrate against flow, an effect that is abrogated by blocking the CXCL12 receptor CXCR4. Once the arteries reach a mature morphology, Dach1 expression is decreased, presumably decreasing its progrowth effects. This decrease is elicited specifically by high, laminar shear stress, suggesting that the changing quality of flow present at the end of 

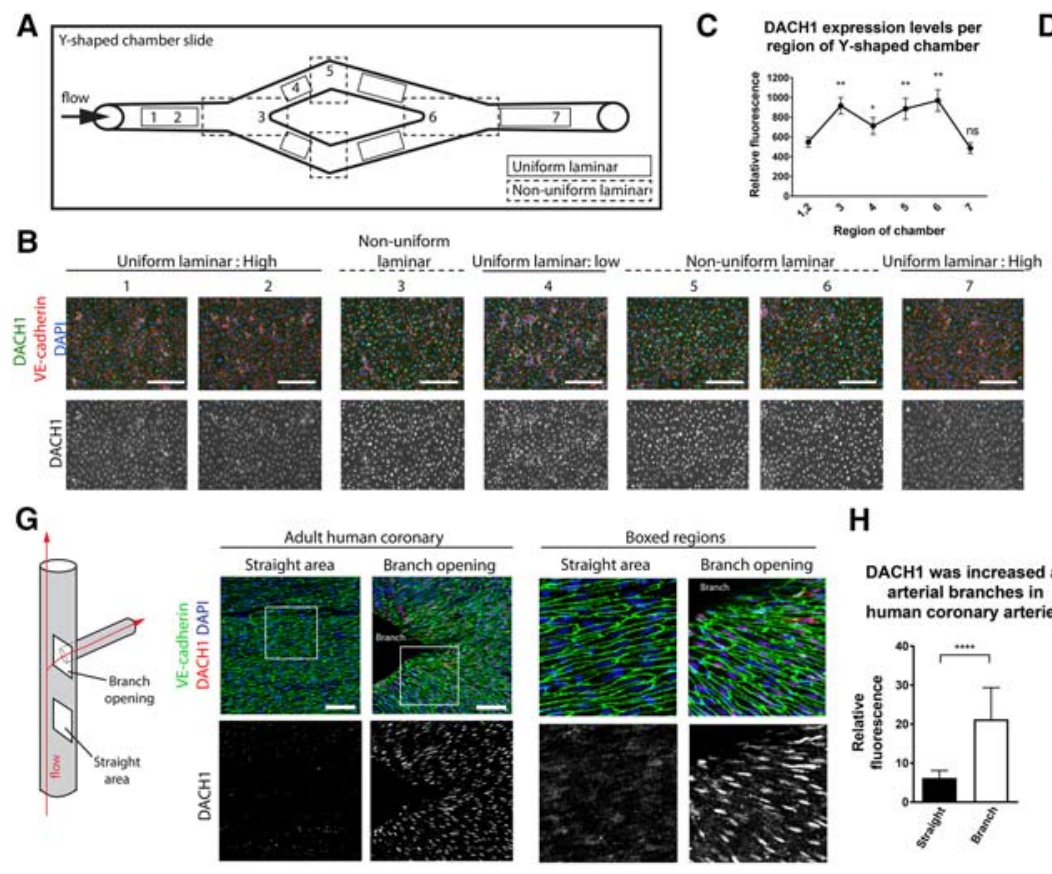

H
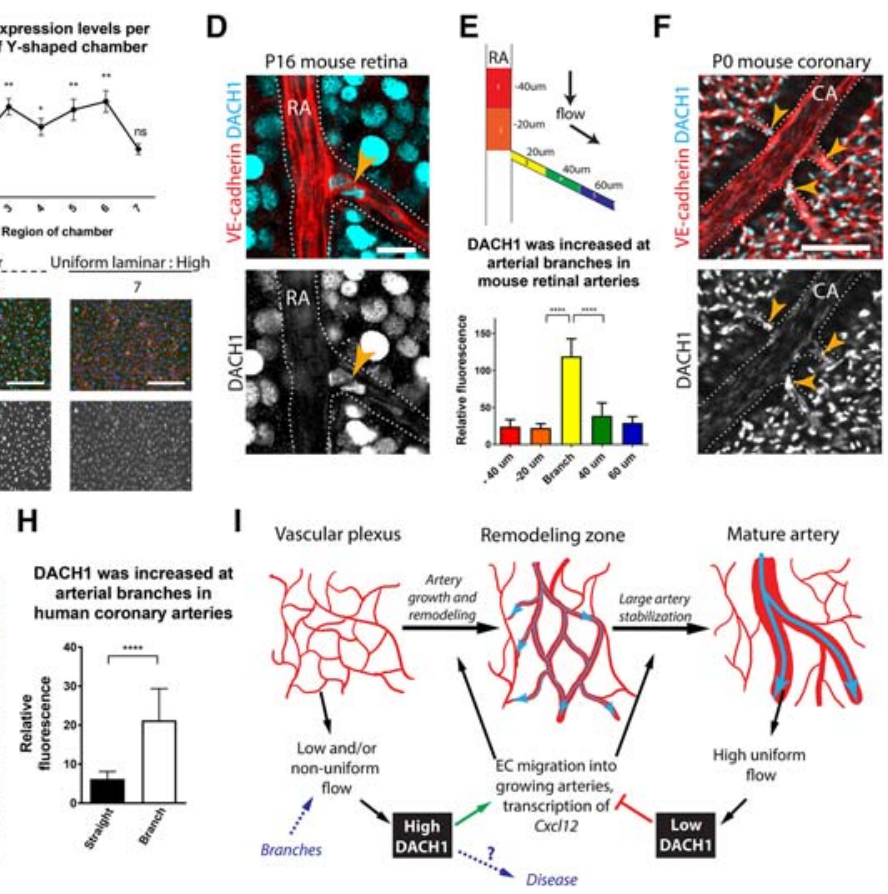

Figure 7. DACH1 is high at branch points in vitro and in mouse and human arteries. $(A)$ Schematic of the Ibidi Y-shaped chamber slide. Branch points create regions that experience uniform (boxes) and nonuniform (dashed-line boxes) laminar shear stresses. (B) Images corresponding to the regions indicated in the schematic. Sites experiencing nonuniform or low shear stress increased nuclear DACH1. $(C)$ Quantification of nuclear DACH1 levels at individual sites along the chamber from two independent experiments. $P$-values were compared with regions 1 and 2. $(D, E)$ Immunofluorescence of retinal arteries (RA; dotted lines) of the postnatal eye $(D)$ and quantification of fluorescence levels $(E)$ showed that DACH1 was significantly higher in endothelial cells specifically at branch points (orange arrowheads in $D$; yellow column in $E$ ). $n=10$ branches from two animals. $(F)$ Immunofluorescence of P0 coronary arteries (CA; dotted lines) showing that DACH1 was up-regulated at branch points (orange arrowheads). $(G, H)$ DACH1 was increased at branch points in human coronary arteries. $(G)$ En face preparations imaged as shown in the schematic, with the boxed regions in the right panels. $(H)$ Quantification of DACH1 fluorescence. $n=8$ straight FOVs from four human coronary arteries; $n=16$ branch FOVs from four human coronary arteries. (I) Working model for the interaction between blood flow and DACH1 expression during arterial remodeling. (EC) Endothelial cell. Error bars indicate standard deviation. (ns) Nonsignificant; $\left(^{*}\right) P<0.05$; $\left(^{* *}\right) P<0.01$; (****) $P<0.0001$. Bars: $B, 200 \mu \mathrm{m} ; D, 10 \mu \mathrm{m} ; F, G, 100 \mu \mathrm{m}$.

arterial remodeling signals the transition into a maturation phase through regulating Dach1 expression. Thus, DACH1 is a transcriptional mediator that interacts with mechanical signals imparted by blood flow to shape the coronary vasculature system (Fig. 7I).

The cellular and molecular mechanisms underlying developmental arterial remodeling remain poorly understood, particularly those that interact with blood flowinduced mechanical signals. Time-lapse analyses of endothelial cells in the yolk sac and regenerating zebrafish fins have shown that endothelial cells migrate against the direction of blood flow into the growing arteries (Sato et al. 2010; Udan et al. 2013; Xu et al. 2014). Although we cannot perform live imaging, our studies on endothelial cell proliferation and polarization suggest that coronary arteries grow in a similar manner. In the developing coronary vasculature, cell division occurs primarily in the immature capillary plexus. Proliferation is decreased in the arterial remodeling zone and absent once the vessels fully transform into an artery, yet the vessel continues to grow in size. This low proliferation, along with data showing that a majority of endothelial cells at the arterial remodeling zone is polarized against flow, suggests that migration provides the additional cells needed for vessel expansion. Animals deficient in endothelial Dach1 had delayed arterial remodeling and polarization toward the artery, providing evidence that the small artery caliber is due to a defect in the migration of endothelial cells from the plexus. Further evidence for such a model is provided by experiments overexpressing Dach1 in endothelial cells, which dramatically increased their alignment, polarization, and migration against flow. These data show that DACH1 facilitates endothelial cell responses to shear stress, which is likely an important component of the arteriogenesis process.

Our data also indicate that $\mathrm{DACH} 1$ induces cell migration through its influence on Cxcl12 expression. Cxcl12 expression was found in cardiomyocytes and developing arterial endothelial cells. Dach1 knockout animals had decreased endothelial expression, while cardiomyocyte expression was still high. In vitro, Dach1 induced Cxcl12 expression, and inhibiting the CXCR4-CXCL12 signaling axis inhibited the aforementioned Dach1-induced migration against flow. CXCL12 is known to be an attractive signal for endothelial cells (Strasser et al. 2010; Bussmann et al. 2011). This is also the case in the 
coronary vasculature. Here, the ligand is highly expressed around the outflow tract, where it attracts coronary plexus cells to the aorta to form the coronary artery stem (Ivins et al. 2015). We show that arterial endothelial cells in the heart also express Cxcl12 in a Dach1-dependent manner and propose that CXCL12 is part of the signal stimulating endothelial cells to migrate against flow into developing arteries. How a secreted ligand provides directional information in a cell-autonomous manner remains an open question. CXCL12 could be tethered to the cell surface in a polarized manner to provide positional information. Alternatively, CXCR4 signaling could be permissive to other directional cues. In zebrafish, a CXCL12 gradient is generated from a constitutive source by differential expression of its alternative receptor, CXCR7 (Venkiteswaran et al. 2013). It would be interesting to investigate any polarized expression of the two CXCL12 receptors in endothelial cells exposed to shear stress. In summary, DACH1 is upstream of the CXCL12/CXCR4 axis, a signaling pathway previously established to regulate endothelial migration during vascular development.

One of the most fascinating aspects of vascular biology is that endothelial cells are highly sensitive to different fluid flow patterns (Dolan et al. 2012), including variations in magnitude and direction. Uniform laminar shear stress found in straight vessel segments initiates an atheroprotective response, while nonuniform flow, such as at arterial branch points, activates inflammatory cascades ( $\mathrm{Li}$ et al. 2005). Endothelial cells can also sense whether flow is parallel or perpendicular to the long axis of their cell body (Wang et al. 2013). Furthermore, accelerating gradients in shear stress elicit responses that are not seen in decelerating gradients of the same magnitude (Szymanski et al. 2008; Dolan et al. 2011; Ostrowski et al. 2014). As such, it is clear that endothelial cells distinguish between different types of shear stress, but how they recognize and respond in a manner that shapes artery development is poorly understood. Our data suggest that shear stress profiles organize artery growth and stabilization through their differential regulation of Dach1. Flow patterns change dramatically as the highly branched vascular plexus is transformed into a linear artery (Bernabeu et al. 2014). Specifically, shear stress changes from low and variable to higher and uniformly laminar. Our in vitro data showed that high, uniformly laminar flow specifically down-regulates Dach1 expression. This suggests that endothelial cells sense changing flow and behave in a manner that supports artery growth and stabilization through regulation of Dach1. Further understanding of these mechanisms linking blood flow with artery development could identify new methods of stimulating arterial regeneration in circumstances where blood flow is compromised, such as in coronary artery disease (Hahn and Schwartz 2009).

One consequence of endothelial cells' ability to sense and respond to differential shear stress profiles is the activation of pathological signaling pathways at specific sites along the artery. Low and/or nonuniform shear stress at arterial branch points and curves stimulates sustained expression of progrowth and inflammatory genes contribut- ing to vascular diseases such as atherosclerosis (Hahn and Schwartz 2009; Dolan et al. 2012). In adult arteries, high flow rates are atheroprotective in part because they induce the transcription factor KLF2, which suppresses genes that stimulate immune cell infiltration, such as Vcam1 and E-selectin (SenBanerjee et al. 2004; Atkins et al. 2008). Conversely, low and nonuniform shear stress activates NF-kB-mediated transcription of inflammatory genes, thus contributing to the preferential accumulation of atherosclerotic plaques at branch points and curves (Gareus et al. 2008). As a result of its suppression specifically by uniform laminar shear stress, DACH1 expression persists at arterial branch points in both mouse and human vessels. Given its progrowth function during development, continued expression of DACH1 at branch points may modulate pathological endothelial cell activation and disease. Indeed, one of the most highly induced genes in Dach1-overexpressing cells was Vcam1 (Supplemental Table 2), which plays a direct role in promoting monocyte migration during atherogenesis and contributes to atherosclerotic plaque expansion (Cybulsky et al. 2001). Future experiments are required to identify a specific role of Dach1 during arterial pathologies, but further dissection could lead to a better understanding of the cellular dynamics of regeneration and arterial disease.

\section{Materials and methods}

Animals

Mouse husbandry and experimentation followed Stanford University Institution Animal Care and Use Committee (IACUC) guidelines. For DACH1 expression analyses, CD1 (Charles River) (Fig. 2B-D') and C57Bl/6.129 (Fig. 3A) mice were used. Dach1 knockout animals were maintained on a C57Bl/6 background and have been described previously (Davis et al. 2001). Control animals for all Dach1 knockout analyses were wild-type C57Bl/ 6 littermates. The Dach1 flox mouse line was generated using

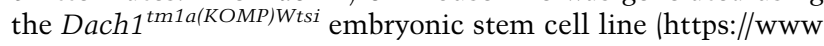
.komp.org/ProductSheet.php? cloneID=689703) sourced from the Knockout Mouse Project (KOMP) Repository at University of California at Davis and subsequently injected into C57Bl/6 blastocysts by the Stanford Transgenic Mouse Research Facility. Once germline transmission was achieved, the Dach1 ${ }^{\text {tm1a(KOMP)Wtsi }}$ allele was crossed to mice with global Flp recombinase expression [The Jackson Laboratory, B6.129S4-Gt(ROSA)26Sortm1(FLP1) Dym/RainJ] to create a conditional knockout allele of Dach1 in which exon 2 is flanked by loxP sites. The following genotyping primers were used to distinguish between wild-type and Dach1 flox alleles: forward, 5'-CTCCTGAAGATGAGGAGCTCACCC $-3^{\prime}$; and reverse, 5'-AACAATTCTTGTCCTTCACGTGCCC-3'. Tie2Cre [The Jackson Laboratories, B6.Cg-Tg(Tek-cre)12Flv/J] was used to create mice with endothelial Dach1 deletion. For experiments, Tie2Cre; Dach1 flox/flox or Tie2Cre; Dach1 $1^{\text {flox } /+}$ male mice were crossed with Dach $1^{\text {flox/flox }}$ females, which were on a mixed background. Control animals for all Dach1 eCKO analyses were Cre-negative littermates.

Human tissue collection

Tissue collection and examination were approved for use in research by the Stanford University Institutional Review Board. Coronary arteries were harvested from diseased human hearts 
that were explanted at the time of cardiac transplantation or from healthy donor hearts that were contraindicated for cardiac transplantation. Hearts were arrested with antegrade cold cardioplegia, surgically explanted, and transported in sterile saline and ice. The mid and distal left anterior descending artery, circumflex coronary artery, and posterior descending artery were dissected from the heart and prepared for histologic analyses as described below.

\section{Immunofluorescence and imaging}

Staged embryonic hearts obtained by timed pregnancies (morning plug designated E0.5) or adult arterial vessels (mouse or human) were dissected and fixed in $4 \%$ paraformaldehyde, washed, and stored at $4^{\circ} \mathrm{C}$ in phosphate-buffered saline (PBS). Whole-mount fluorescence microscopy was performed on intact hearts or adult arteries that had been cut and opened lengthwise. Staining was performed in $1.5-\mathrm{mL}$ tubes subjected to constant rotation. Primary antibodies were diluted in blocking solution $15 \%$ goat serum, $0.5 \%$ Triton X-100 in PBS) and incubated with tissues overnight at $4{ }^{\circ} \mathrm{C}$. Tissues were then washed with PBT (PBS with $0.5 \%$ Triton X-100) four times for $1 \mathrm{~h}$ each before another overnight incubation with secondary antibodies diluted in blocking solution. Specimens were then washed again, placed in VectaShield (Vector Laboratories, H-1000), and imaged using an inverted Zeiss LSM700 confocal microscope. Embryonic hearts were oriented in eight-well chambered coverglasses (ThermoFisher Scientific, 155409) to invariably image the left and right coronary arteries; adult arteries were placed on microscope slides en face. Images were digitally captured and processed using the Zeiss Zen software (2011).

The following primary antibodies were used: VE-cadherin (1:100; BD Biosciences, 550548), anti-DACH1 (1:500; Proteintech 10914-1-AP), anti-SM-MHC (1:300; Biomedical Technologies, BT-562), anti-VEGFR2 (1:125; R\&D Systems, AF644), antiJAG1 (1:125; R\&D Systems, AF599), anti-ERG (1:500; Abcam, ab92513), and anti-GOLPH4 (1:500; Abcam, ab28049). Secondary reagents were Alexa fluor-conjugated antibodies $(405,488,555$, 633) from Life Technologies used at 1:250. DAPI was obtain from Sigma-Aldrich (D9542) and used at $2 \mu \mathrm{g} / \mathrm{mL}$.

Quantification of Dach1 knockout and Dach1 eCKO phenotypes

Coronaryarterysize Endothelial cells that line the interior layer of artery lumens in E17.5 hearts were immunostained using VE-cadherin-specific antibodies and imaged using whole-mount confocal microscopy. For Figures $1 \mathrm{D}$ and $3 \mathrm{C}$, the diameter of the main (primary branch) coronary artery was measured at five locations $(200,300,400,500$, and $600 \mu \mathrm{m}$ from the first appearance of the artery near the aorta) and averaged for both the right and left coronary arteries $(n=9$ wild type, $n=28$ Dach 1 heterozygous, $n=$ 14 Dach1 knockout; $n=32$ control, $n=18$ Dach1 eCKO). For Figure $1 \mathrm{~F}$, one measurement each for the diameter of the primary, secondary, and tertiary branches of the left and right coronary arteries was collected. Values for each branch type were averages from 13 wild-type and 14 knockout hearts. Average diameters were plotted, and linear regression lines were calculated between the primary, secondary, and tertiary branches to determine the slopes. For Figure 1G, looping was defined as an artery that branches away from and then reconnects with an artery in a distal location (see Fig. 1A, arrowheads).

Coronary plexus properties Vessel coverage (Fig. 1B; Supplemental Fig. S3D) and junction density (Fig. 1C; Supplemental Fig. S3E) were quantified for E17.5 (Fig. 1) and E15.5 (Supplemental Fig.
S3) hearts, respectively, using the Angiotool program (Zudaire et al. 2011). The analyses were conducted on a $375-\mu \mathrm{m} \times 375$ $\mu \mathrm{m}$ region from a single optical plane of the left intramyocardial endothelial plexus at the same or similar depth. At E17.5, nine wild-type and 14 knockout animals were analyzed. At E15.5, 13 wild-type and 12 knockout animals were analyzed.

Coronary smooth muscle coverage The smooth muscle surrounding the developing coronary artery was immunolabeled for SMMHC. To quantify the area covered by the smooth muscle cells, a region of the left coronary artery with an approximate length of $550 \mu \mathrm{m}$ for E17.5 and $450 \mu \mathrm{m}$ for E15.5 was cropped out from a maximal projection, excluding the surface optical planes. The area of the cropped-out region was then quantified to determine the total area of the artery. The same thresholding parameter was then applied to the SM-MHC-labeled channel to quantify the area covered by smooth muscle. A final percentage of coverage was obtained by dividing total area by covered area. For E17.5 Dach1 knockout (Fig. 1E), eight wild-type and 10 Dach1 knockout animals were analyzed. For E17.5 Dach1 eCKO (Fig. $3 \mathrm{D}), 11$ control and seven $e C K O$ animals were analyzed. For E15.5 (Supplemental Fig. S3F), 13 wild-type and 12 Dach1 knockout animals were analyzed.

Remodeling zone maturation Whole-mount images of hearts immunostained with either VE-cadherin and JAG1 or VE-cadherin alone were processed to display the arterial remodeling zone of the developing right coronary artery at E13.5, E14.5, E15.5, and E17.5. The remodeling zone was classified as early, late, or mature according to the extent of branching as well as the presence and diameter of an emerging dominant artery (see Fig. 3E). The numbers of early, late, and mature remodeling zones were counted for wild-type and Dach1-deficient hearts. $N$-values are shown in Figure 3F.

Proliferation rates in developing hearts To assess cell proliferation, mitotically active cells were measured through the incorporation of EdU. Pregnant C57Bl/ 6 females with E14.5 embryos received a single intraperitoneal injection of $400 \mu \mathrm{g}$ of EdU dissolved in 200 $\mu \mathrm{L}$ of dimethyl sulfoxide (DMSO). Three hours later, embryonic hearts were dissected, fixed, and immunostained for VE-cadherin and ERG as described above. Subsequently, EdU incorporation into DNA was detected through the Click-iT EdU Alexa fluor 555 imaging kit (ThermoFisher Scientific, C10338) performed at room temperature using the protocol recommended by the manufacturer. Confocal $z$-stacks at 20x magnification (with 3$\mu \mathrm{m}$ intervals between $z$-planes) through the right lateral side of each heart were collected. A $200-\mu \mathrm{m} \times 200-\mu \mathrm{m}$ square was drawn around the vascular plexus on the heart surface (subepicardial) and within the myocardium (intramyocardial), at a coronary artery remodeling zone, and a mature artery. Representative images of cell proliferation in different vessel compartments are shown in Figure 2F. $\mathrm{ERG}^{+}$and $\mathrm{EdU}^{+}$double-positive endothelial cells (Fig. 2F, purple cells, arrowheads) were highlighted using the Image Colocalization plug-in. For quantification in Figure $2 \mathrm{G}$, all $\mathrm{ERG}^{+}$cells were counted using the Cell Counter plug-in from ImageJ, and the percentage of those also EdU ${ }^{+}$was considered proliferative. The data were compiled from four E14.5 C57Bl/6 wild-type hearts.

Polarity quantification in developing hearts Endothelial cell polarity in developing coronary arteries was determined by measuring the Golgi (GOLPH4) position relative to the nuclei (ERG) and sorting the cells into an "against flow," "static," or "with flow" category based on their angle relative to the local blood flow direction. 
Figure 2, H and I, was quantified from four wild-type arteries. Figure $3, \mathrm{G}$ and $\mathrm{H}$, was quantified from seven control and nine Dach1 eCKO arteries.

Echocardiography Left ventricular geometry and function were evaluated in 3 - to 5 -mo-old mice $(n=8$ wild type; $n=8$ Dach1 $e C K O$,) with a high-resolution (30 MHz) Vevo 2100 transthoracic echocardiography system (VisualSonics). Chest hair was removed with depilatory cream, and mice were sedated with $1 \%-3 \%$ inhaled isoflurane titrated to a heart rate between 350 and 450 per minute. Images were obtained through a parasternal short axis view with M-mode ultrasound at the level of the papillary muscle and midway between the papillary muscle and apex. Ejection fraction, fractional shortening, and internal ventricular dimensions were computed with the Vevo 770 standard measurement package. All analyses were performed by a single investigator blinded to the genotype.

\section{Quantification of Dach1 fluorescence}

Expression in the developing hearts DACH1 expression levels in capillary vessels and developing arteries (Fig. $\left.2 \mathrm{~B}^{\prime}-\mathrm{D}^{\prime}\right)$ were quantified from confocal images of hearts immunostained for VE-cadherin and DACH1. Expression in capillary, remodeling zone, and arterial vessels was quantified from the same $z$-plane by selecting a line segment along the endothelial cells of each vessel type in ImageJ. Next, average fluorescence intensity was calculated for each segment. $N=6$ hearts per time point, and VE-cadherin served as a control.

Expression under in vitro flow DACH1 levels were quantified by calculating fluorescence intensity within the nucleus using ImageJ. To delineate the nucleus, DAPI-positive regions were selected using the thresholding function, and DACH1 fluorescence in this region was measured. Background fluorescence was calculated by averaging the fluorescence intensity of five cell-free regions and subtracted from the DACH1 intensity values. Each experiment was replicated three times, in which two or more fields of view (FOVs) were quantified for each treatment (parallel plate) or region (impinging flow, Y-shaped chamber).

Expression at arterial branch points For mouse tissues, P7 through adult retinas were fixed and stained for confocal imaging. Optical sections through the tissue were taken for each retina, and the images were subsequently analyzed using ImageJ. Vessel segments around the branch point were divided into five separate $20-\mu \mathrm{m}$ long regions of different distances from the branch point for measurements. Within each segment, a line was drawn though endothelial cell nuclei, and the maximum DACH1 intensity was recorded. Fifty-eight total branches were analyzed $(n=25$ from three animals at $\mathrm{P} 7 / \mathrm{P} 9 ; n=10$ from two animals at P19; and $n=$ 23 from two adult animals).

For human arteries, the left anterior descending artery, the left circumflex artery, and the right coronary artery from pediatric $(n=1)$ and adult $(n=3)$ donors were fixed, opened lengthwise, and stained for confocal imaging as described above. Stained arteries were opened and oriented lumen side up on a microscope slide for en face imaging. For quantification, a total of two $200-\mu m^{2}$ FOVs from each of eight separate branch points $(n=1-3$ branch points per donor) and four straight regions ( $n=1$ per donor) were imaged using the same settings and analyzed for fluorescence intensity as described above (see "Expression Under In Vitro Flow").

\section{Quantification of Cxcl12 expression}

To examine Cxcl12 expression levels in vivo, we bred the Dach1 knockout mice with a Cxcl12 reporter line where DsRed replaces the endogenous $C x c 112$ gene and generated wild-type, Dach1 heterozygous, and Dach1 knockout embryos under a Cxcl12-DsRed background as seen in Figure 5, C and D. Note that these mice were also heterozygous for the Cxcl12 gene. Confocal images of hearts with endogenous expression of DsRed (a readout of Cxcl12 expression) were used for quantification. Expression in capillary, remodeling zone, and arterial vessels were quantified from the same $z$-planes (maximum projection of four optical sections) by selecting three $50-\mu \mathrm{m}$ line segments along the endothelial cells of each vessel type in ImageJ. Next, average fluorescence intensity was calculated for each segment, which was normalized to the wild-type capillary vessels within litters. $n=4$ Dach1 wild type, $n=8$ Dach1 heterozygous, and $n=5$ Dach1 knockout, all containing one copy of the Cxcl12-DsRed allele.

\section{Cxcl12 in situ hybridization}

In situ hybridization on paraffin sections was performed as described previously (de la Pompa et al. 1997). Briefly, E16.5 control and Dach1 eCKO embryos ( $n=9$ control; $n=5$ Dach1 eCKO) were dissected in $1 \times$ PBS and fixed in $4 \%$ PFA overnight at $4{ }^{\circ} \mathrm{C}$. Fixed embryos were embedded in paraffin and sectioned at 10 $\mu \mathrm{m}$ thickness.

Slides were hybridized overnight with $1 \mu \mathrm{g} / \mathrm{mL}$ antisense Cxcl12 digoxigenin-labeled probe at $65^{\circ} \mathrm{C}$. After washing in salt sodium citrate (SSC) buffer, slides were incubated with alkaline phosphatase-coupled anti-digoxigenin antibodies overnight. BM-purple (Sigma-Aldrich, 11442074001) was used to develop the color in the dark to the desired extent. Slides were then fixed in $4 \%$ PFA for $20 \mathrm{~min}$, dehydrated through ethanol series and xylene (Sigma-Aldrich, 534056), and mounted using Entellan (Electron Microscopy Sciences, 14800). Images were acquired using the Zeiss Axio imager.A2 upright microscope.

\section{Dachl depletion in primary endothelial cell cultures}

Lentiviral introduction of shRNAs specific for either a control gene $\left(\mathrm{BCR}_{\text {; }}\right.$ Mission shRNA, Sigma-Aldrich, SHCLNGNM_001081412) or Dach1 (Dach1; Mission shRNA, Sigma-Aldrich, SHCLNG-NM_004392) were used to knock down mRNA in HCAECs (Lonza). shRNA-expressing lentiviral particles were prepared by transfecting (Lipofectamine 2000, Thermo Fisher Scientific) VSVG, delta8.2, and Sigma Mission shRNA plasmids into HEK293T cells in DMEM with penicillin/streptomycin and $10 \%$ FBS. Viral particles were collected after $48 \mathrm{~h}$ and then mixed with polybrene (to increase infectivity) and EGM2-MV medium before being added to HCAECs. Infected cells were selected using a puromycin selection step. Knockdown specifically in Dach1 shRNA was analyzed by immunofluorescence and qPCR (Supplemental Fig. S3H,I).

Migration of HCAECs (Fig. 3I) was performed using Falcon cell culture inserts with 8 - $\mu \mathrm{m}$ pores (Corning, 353097). The bottom of the inserts was precoated with fibronectin (1:100; Sigma, F0895) for at least $1 \mathrm{~h}$ prior to the start of the experiment. Endothelial cells $\left(5 \times 10^{4}\right.$ cells) were used $48 \mathrm{~h}$ after lentiviral shRNA infections were suspended in EBM2 basal medium (Lonza) and added to the tops of the inserts. Each insert was then placed in one well of a 24-well plate containing $500 \mu \mathrm{L}$ of EGM2-MV medium. Cells were cultured for $3.5 \mathrm{~h}$ at $37^{\circ} \mathrm{C}$ and $5 \% \mathrm{CO}_{2}$. Cells were fixed with $4 \%$ paraformaldehyde (Electron Microscopy Sciences) for $20 \mathrm{~min}$, permeabilized with $100 \%$ methanol for $20 \mathrm{~min}$ at room temperature, and stained with $0.1 \%$ Crystal Violet acetate. 
The cells remaining on top of the insert membrane were removed using a cotton swab, after which those that migrated across to the bottom were imaged at $71 \times$ magnification on a stereomicroscope (Zeiss SteREO Discovery.V20). For quantification, the total number of cells in five comparable FOVs for each condition was counted. The entire experiment was repeated three times.

Exposure of endothelial cells to shear stress

Parallel plate and Y-shaped chamber HCAECs (not more than passage 8) were plated at $90 \%$ confluency in each lane of either a $\mu$-Slide $\mathrm{VI}^{0.4}$ (Ibidi, 80601) or $\mu$-Slide Y-shaped (Ibidi, 80121) chamber coated with fibronectin (1:100; Sigma, F0895) $2 \mathrm{~h}$ prior to the experiment. Culture medium consisted of Leibowitz's L-15 medium without phenol red (ThermoFisher Scientific, 21083-027) supplemented with EGM2-MV SingleQuot kit supplement and growth factors (Lonza, CC-4147). Uniform laminar shear stress (continuous at 5,20 , or 35 dynes $/ \mathrm{cm}^{2}$ ) was created by attaching slide chambers to an Ibidi pump system (Ibidi, 10902) mounted on an inverted microscope (Zeiss Axio Observer.Z1) fitted with a heated Plexiglas enclosed incubator. Time-lapse images were taken every $15 \mathrm{~min}$, starting $30 \mathrm{~min}$ after pump initiation. The experiment was also performed at 20 dynes $/ \mathrm{cm}^{2}$ using pulsatile flow. For CXCR4 inhibition experiments, $10 \mu \mathrm{M}$ AMD3100 was added to the medium prior to the experiment $(t=0 \mathrm{~h})$ and mixed in with L15 and added again at $48 \mathrm{~h}$ into the experiment. A separate fluidic unit supplied medium without AMD3100 for the control cells. All parallel plate experiments were repeated three times. The Y-shaped chamber experiment was repeated twice.

Orbital shaker In order to collect the required number of cells for qPCR and Western analyses, an orbital shaker was used to generate laminar shear stress as described previously (Dardik et al. 2005). Endothelial cells were plated in six-well plates and placed on an orbital shaker set at $210 \mathrm{rpm}$, which recapitulates a shear stress of $\sim 14$ dyne $/ \mathrm{cm}^{2}$. Cells were subjected to rotation for 24$48 \mathrm{~h}$ before RNA was collected using a Qiagen RNeasy minikit (Qiagen, 74104) and used in qPCR experiments (details below in "qPCR Analysis"). In some experiments, protein lysates were also collected for subsequent Western analyses.

Impinging jet The impinging flow device has been described previously (Ostrowski et al. 2014) and consisted of a source of jetted medium mounted on a motorized microscope stage within a temperature-controlled cabinet. The device was suspended at a fixed height above a glass-bottom six-well cell culture dish (six-well no. 1.5 glass-bottom dishes; In Vitro Scientific) coated with $0.2 \%$ gelatin (Sigma-Aldrich) containing confluent layers of HCAECs. A peristaltic pump was used to deliver cell culture medium consisting of Leibowitz's L-15 medium without phenol red (ThermoFisher Scientific, 21083-027) supplemented with EGM2MV SingleQuot kit supplement and growth factors (Lonza, CC4147) perpendicular to the cell surface at $3 \mathrm{~L} / \mathrm{min}$. Cells were maintained under constant impinging for $24 \mathrm{~h}$ before being fixed, immunostained for DACH1, and quantified as described above. $n=14$ FOVs per position from seven experiments.

Overexpression of Dach1 in human coronary endothelial cells

For Dach1 overexpression experiments, pCMV6 mouse Dachshund1 GFP-tagged (transcript variant 1, OriGene Technologies, MG216327) was purchased, and the Dach1-coding region was subcloned into a lentiviral-ready $p L J M-e G F P$ plasmid (Addgene, plasmid 19319). Lentiviral particles were produced by transfection (Lipofectamine 2000, Thermo Fisher Scientific) of the
pLIM1-Dach1-eGFP (or pLJM1-eGFP for control), VSVG, and psPAX2 plasmids into HEK293T cells in DMEM with penicillin/streptomycin and 10\% FBS. Viral particles were collected after $48 \mathrm{~h}$ and mixed with polybrene (to increase infectivity) and EGM2-MV medium before being added to HCAECs. Infected HCAECs were analyzed for transduction efficiency $48 \mathrm{~h}$ following infection by observing GFP fluorescence. Cultures with $>90 \%$ of cells visibly expressing GFP were used for experiments, parallel plate flow chamber, and RNA sequencing. For analysis of HCAEC migration (Figs. 4B,C, 5F), the mTrackJ plug-in of Image was used to follow cell tracks of individual cells from time-lapse images taken under flow conditions (described above). A total of 40 randomly selected cells among four FOVs from each of three experiments (120 migration tracks total) was quantified per condition. The polarity of cells from the in vitro experiments in Figures 4I and 5G was quantified after the flow experiment endpoint at $72 \mathrm{~h}$ by measuring the angle of the line connecting the nuclei and Golgi relative to the flow direction (see the schematic in Fig. 4G). Because the channel of the flow chamber is straight, uniform laminar flow direction is directly parallel from the flow inlet to the flow outlet. Cells with Golgi aligned within $45^{\circ}$ against the flow direction were classified as "against flow," while cells $45^{\circ}$ with the flow direction were classified as "with flow." The remaining cells were placed in the "static" category.

\section{RNA sequencing}

RNA was collected from HCAECs using the RNeasy minikit (Qiagen, 74104). RNA from embryonic hearts at E17.5 was collected using the Arcturus PicoPure RNA isolation kit (Applied Biosystems, 12204-01). RNA quantity and quality (RNA integrity number score) were verified via RNA Qubit and the Agilent Bioanalyzer QC service provided by the Stanford Protein and Nucleic Acid (PAN) Facility. The cDNA libraries were then created from the RNA using the NEBNext Ultra Directional RNA library preparation kit for Illumina (New England Biolabs, E7420S) following the manufacturer's instructions. The cDNA libraries were checked for quality by DNA Qubit and the Bioanalyzer QC. Subsequently, they were sent for Illumina sequencing at the Stanford Center for Genomics and Personalized Medicine (SCGPM) (paired end, HiSeq 2000, 101 bases twice). The RNA sequencing reads were first aligned to the reference genome $(\mathrm{mm} 9$ for mice and hg19 for humans) using the Burrows-Wheeler Alignment (BWA) tool by the SCGPM. Read counting and differential gene expression were analyzed through the Bioconductor (3.2) package rnaseqGene in R, which uses DESeq2. $P$-adjusted values were calculated using the Benjamini-Hochberg adjustment, with the FDR value of $0.1 \%$ as the cutoff. GO analysis was analyzed using the Bioconductor package goseq (1.22.0). A subset of the differentially expressed genes found in the data set was subsequently validated by qPCR.

\section{qPCR analysis}

RNA was isolated with the RNeasy Plus minikit from Qiagen (74104). Two-hundred nanograms of RNA was used for the RT reaction using the iScript RT mix from Bio-Rad (1708840). qPCR was performed using the iTaq Universal SYBR Green Supermix from Bio-Rad (1725121) and run on a CFX96 Touch real-time PCR detection system. Primers were designed and ordered from IDT using the PrimerQuest real-time PCR design tool.

\section{Acknowledgments}

We thank Dr. Graeme Mardon for the Dach1 knockout mouse strains, and the Red-Horse laboratory members for their valuable 
input and insightful comments. A.H.C. is funded by an American Heart Association Predoctoral Fellowship (15PRE23020030). K.R.-H. is supported by the National Institutes of Health (RO1HL128503) and the New York Stem Cell Foundation (Robertson Investigator). A.H.C. and K.R.-H. designed the study, performed experiments and data analysis, and wrote the manuscript. B.C.R., H.I.C., G.D., and A.P. performed experiments and assisted in editing the manuscript. V.N.S., G.G.F., and A.R.D. assisted in performing impinging flow experiments and provided guidance on fluid mechanics and shear stress modeling. A.B.G. and J.W. assisted in the acquisition of human coronary samples.

\section{References}

Ara T, Tokoyoda K, Okamoto R, Koni PA, Nagasawa T. 2005. The role of CXCL12 in the organ-specific process of artery formation. Blood 105: 3155-3161.

Atkins GB, Wang Y, Mahabeleshwar GH, Shi H, Gao H, Kawanami D, Natesan V, Lin Z, Simon DI, Jain MK. 2008. Hemizygous deficiency of Krüppel-like factor 2 augments experimental atherosclerosis. Circ Res 103: 690-693.

Bernabeu MO, Jones ML, Nielsen JH, Krüger T, Nash RW, Groen D, Schmieschek S, Hetherington J, Gerhardt H, Franco CA, et al. 2014. Computer simulations reveal complex distribution of haemodynamic forces in a mouse retina model of angiogenesis. J R Soc Interface 11: 20140543.

Bussmann J, Wolfe SA, Siekmann AF. 2011. Arterial-venous network formation during brain vascularization involves hemodynamic regulation of chemokine signaling. Development 138: $1717-1726$.

Cavallero S, Shen H, Yi C, Lien C-L, Kumar SR, Sucov HM. 2015. CXCL12 signaling is essential for maturation of the ventricular coronary endothelial plexus and establishment of functional coronary circulation. Dev Cell 33: 469-477.

Chen HI, Poduri A, Numi H, Kivelä R, Saharinen P, McKay AS, Raftrey B, Churko J, Tian X, Zhou B, et al. 2014a. VEGF-C and aortic cardiomyocytes guide coronary artery stem development. J Clin Invest 124: 4899-4914.

Chen HI, Sharma B, Akerberg BN, Numi HJ, Kivelä R, Saharinen $\mathrm{P}$, Aghajanian H, McKay AS, Bogard PE, Chang AH, et al. $2014 \mathrm{~b}$. The sinus venosus contributes to coronary vasculature through VEGFC-stimulated angiogenesis. Development 141: 4500-4512.

Corti P, Young S, Chen C-Y, Patrick MJ, Rochon ER, Pekkan K, Roman BL. 2011. Interaction between alk 1 and blood flow in the development of arteriovenous malformations. Development 138: 1573-1582.

Culver JC, Dickinson ME. 2010. The effects of hemodynamic force on embryonic development. Microcirculation 17: 164-178.

Cybulsky MI, Iiyama K, Li H, Zhu S, Chen M, Iiyama M, Davis V, Gutierrez-Ramos JC, Connelly PW, Milstone DS. 2001. A major role for VCAM-1, but not ICAM-1, in early atherosclerosis. J Clin Invest 107: 1255-1262.

Dardik A, Chen L, Frattini J, Asada H, Aziz F, Kudo FA, Sumpio BE. 2005. Differential effects of orbital and laminar shear stress on endothelial cells. J Vasc Surg 41: 869-880.

Davis RJ, Shen W, Sandler YI, Amoui M, Purcell P, Maas R, Ou CN, Vogel H, Beaudet AL, Mardon G. 2001. Dach1 mutant mice bear no gross abnormalities in eye, limb, and brain development and exhibit postnatal lethality. Mol Cell Biol 21: 1484-1490.

de la Pompa JL, Wakeham A, Correia KM, Samper E, Brown S, Aguilera RJ, Nakano T, Honjo T, Mak TW, Rossant J, et al.
1997. Conservation of the Notch signalling pathway in mammalian neurogenesis. Development 124: 1139-1148.

Dolan JM, Meng H, Singh S, Paluch R, Kolega J. 2011. High fluid shear stress and spatial shear stress gradients affect endothelial proliferation, survival, and alignment. Ann Biomed Eng 39: 1620-1631.

Dolan JM, Kolega J, Meng H. 2012. High wall shear stress and spatial gradients in vascular pathology: a review. Ann Biomed Eng 41: 1411-1427.

Ehling M, Adams S, Benedito R, Adams RH. 2013. Notch controls retinal blood vessel maturation and quiescence. Development 140: 3051-3061.

Franco CA, Jones ML, Bernabeu MO, Geudens I, Mathivet T, Rosa A, Lopes FM, Lima AP, Ragab A, Collins RT, et al. 2015. Dynamic endothelial cell rearrangements drive developmental vessel regression. PLoS Biol 13: 1-19.

Franco CA, Jones ML, Bernabeu MO, Vion A-C, Barbacena P, Fan I, Mathivet T, Fonseca CG, Ragab A, Yamaguchi TP, et al. 2016. Non-canonical Wnt signalling modulates the endothelial shear stress flow sensor in vascular remodelling. Elife 5: $\mathrm{e} 07727$.

Gareus R, Kotsaki E, Xanthoulea S, van der Made I, Gijbels MJ, Kardakaris R, Polykratis A, Kollias G, de Winther MPJ, Pasparakis M. 2008. Endothelial cell-specific NF- $\kappa B$ inhibition protects mice from atherosclerosis. Cell Metab 8: 372-383.

Gay L, Miller MR, Ventura PB, Devasthali V, Vue Z, Thompson HL, Temple S, Zong H, Cleary MD, Stankunas K, et al. 2013. Mouse TU tagging: a chemical/genetic intersectional method for purifying cell type-specific nascent RNA. Genes Dev 27: 98-115.

Hahn C, Schwartz MA. 2009. Mechanotransduction in vascular physiology and atherogenesis. Nat Rev Mol Cell Biol 10: 53-62.

Harrison MRM, Bussmann J, Huang Y, Zhao L, Osorio A, Burns CG, Burns CE, Sucov HM, Siekmann AF, Lien C-L. 2015. Chemokine-guided angiogenesis directs coronary vasculature formation in zebrafish. Dev Cell 33: 442-454.

Ivins S, Chappell J, Vernay B, Suntharalingham J, Martineau A, Mohun TJ, Scambler PJ. 2015. The CXCL12/CXCR4 axis plays a critical role in coronary artery development. Dev Cell 33: 455-468.

Kalousova A, Mavropoulos A, Adams BA, Nekrep N, Li Z, Krauss S, Stainier DY, German MS. 2010. Dachshund homologues play a conserved role in islet cell development. Dev Biol 348: 143-152.

Li Y-SJ, Haga JH, Chien S. 2005. Molecular basis of the effects of shear stress on vascular endothelial cells. I Biomech 38: 1949-1971.

Lucitti JL, Jones EAV, Huang C, Chen J, Fraser SE, Dickinson ME. 2007. Vascular remodeling of the mouse yolk sac requires hemodynamic force. Development 134: 3317-3326.

Mahadevan A, Welsh IC, Sivakumar A, Gludish DW, Shilvock AR, Noden DM, Huss D, Lansford R, Kurpios NA. 2014. The left-right Pitx2 pathway drives organ-specific arterial and lymphatic development in the intestine. Dev Cell 31: 690-706.

Mardon G, Solomon NM, Rubin GM. 1994. dachshund encodes a nuclear protein required for normal eye and leg development in Drosophila. Development 120: 3473-3486.

Ostrowski MA, Huang NF, Walker TW, Verwijlen T, Poplawski C, Khoo AS, Cooke JP, Fuller GG, Dunn AR. 2014. Microvascular endothelial cells migrate upstream and align against the shear stress field created by impinging flow. Biophys $J$ 106: 366-374. 
Chang et al.

Paz dela NG, Walshe TE, Leach LL, Saint-Geniez M, D'Amore PA. 2012. Role of shear-stress-induced VEGF expression in endothelial cell survival. J Cell Sci 125: 831-843.

Popov VM, Wu K, Zhou J, Powell MJ, Mardon G, Wang C, Pestell RG. 2010. The Dachshund gene in development and hormone-responsive tumorigenesis. Trends Endocrinol Metab 21: 41-49.

Sato Y, Poynter G, Huss D, Filla MB, Czirok A, Rongish BJ, Little CD, Fraser SE, Lansford R. 2010. Dynamic analysis of vascular morphogenesis using transgenic quail embryos. PLoS One 5: e12674.

Schols D, Struyf S, Van Damme J, Esté JA, Henson G, De Clercq E. 1997. Inhibition of T-tropic HIV strains by selective antagonization of the chemokine receptor CXCR4. I Exp Med 186: 1383-1388.

SenBanerjee S, Lin Z, Atkins GB, Greif DM, Rao RM, Kumar A, Feinberg MW, Chen Z, Simon DI, Luscinskas FW, et al. 2004. KLF2 is a novel transcriptional regulator of endothelial proinflammatory activation. J Exp Med 199: 1305-1315.

Simons M, Eichmann A. 2015. Molecular controls of arterial morphogenesis. Circ Res 116: 1712-1724.

Strasser GA, Kaminker JS, Tessier-Lavigne M. 2010. Microarray analysis of retinal endothelial tip cells identifies CXCR4 as a mediator of tip cell morphology and branching. Blood 115: 5102-5110.

Szymanski MP, Metaxa E, Meng H, Kolega J. 2008. Endothelial cell layer subjected to impinging flow mimicking the apex of an arterial bifurcation. Ann Biomed Eng 36: 1681-1689.

Tachibana K, Hirota S, Iizasa H, Yoshida H, Kawabata K, Kataoka Y, Kitamura Y, Matsushima K, Yoshida N, Nishikawa S, et al. 1998. The chemokine receptor CXCR4 is essential for vascularization of the gastrointestinal tract. Nature 393: 591-594.

Takabatake Y, Sugiyama T, Kohara H, Matsusaka T, Kurihara H, Koni PA, Nagasawa Y, Hamano T, Matsui I, Kawada N, et al. 2009. The CXCL12 (SDF-1)/CXCR4 axis is essential for the development of renal vasculature. I Am Soc Nephrol 20: 1714-1723.

Udan RS, Vadakkan TJ, Dickinson ME. 2013. Dynamic responses of endothelial cells to changes in blood flow during vascular remodeling of the mouse yolk sac. Development 140: 4041-4050.

Venkiteswaran G, Lewellis SW, Wang J, Reynolds E, Nicholson C, Knaut H. 2013. Generation and dynamics of an endogenous, self-generated signaling gradient across a migrating tissue. Cell 155: 674-687.

Volz KS, Jacobs AH, Chen HI, Poduri A, McKay AS, Riordan DP, Kofler N, Kitajewski J, Weissman I, Red-Horse K. 2015. Pericytes are progenitors for coronary artery smooth muscle. ed. G. Cossu. Elife 4: e10036.

Wang C, Baker BM, Chen CS, Schwartz MA. 2013. Endothelial cell sensing of flow direction. Arterioscler Thromb Vasc Biol 33: 2130-2136.

Wang S, Helker CSM, Rasouli SJ, Maischein H-M, Offermanns S, Herzog W, Kwon H-B, Stainier DYR. 2016. In vivo modulation of endothelial polarization by Apelin receptor signalling. Nat Commun 7: 1-12.

Xu C, Hasan SS, Schmidt I, Rocha SF, Pitulescu ME, Bussmann J, Meyen D, Raz E, Adams RH, Siekmann AF. 2014. Arteries are formed by vein-derived endothelial tip cells. Nat Commun 5: 5758.

Zudaire E, Gambardella L, Kurcz C, Vermeren S. 2011. A computational tool for quantitative analysis of vascular networks. PLoS One 6: e27385. 


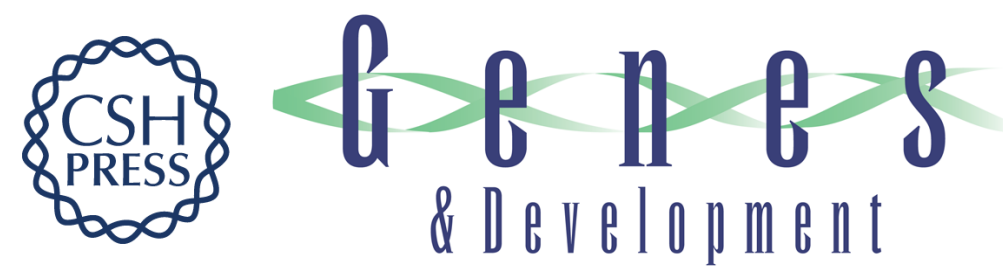

\title{
DACH1 stimulates shear stress-guided endothelial cell migration and coronary artery growth through the CXCL12-CXCR4 signaling axis
}

\author{
Andrew H. Chang, Brian C. Raftrey, Gaetano D'Amato, et al.
}

Genes Dev. 2017, 31: originally published online August 4, 2017

Access the most recent version at doi:10.1101/gad.301549.117

\section{Supplemental http://genesdev.cshlp.org/content/suppl/2017/08/04/gad.301549.117.DC1 Material}

References This article cites 46 articles, 19 of which can be accessed free at: http://genesdev.cshlp.org/content/31/13/1308.full.html\#ref-list-1

Creative This article is distributed exclusively by Cold Spring Harbor Laboratory Press for the first Commons six months after the full-issue publication date (see

License http://genesdev.cshlp.org/site/misc/terms.xhtml). After six months, it is available under a Creative Commons License (Attribution-NonCommercial 4.0 International), as described at http://creativecommons.org/licenses/by-nc/4.0/.

Email Alerting Receive free email alerts when new articles cite this article - sign up in the box at the top Service right corner of the article or click here.

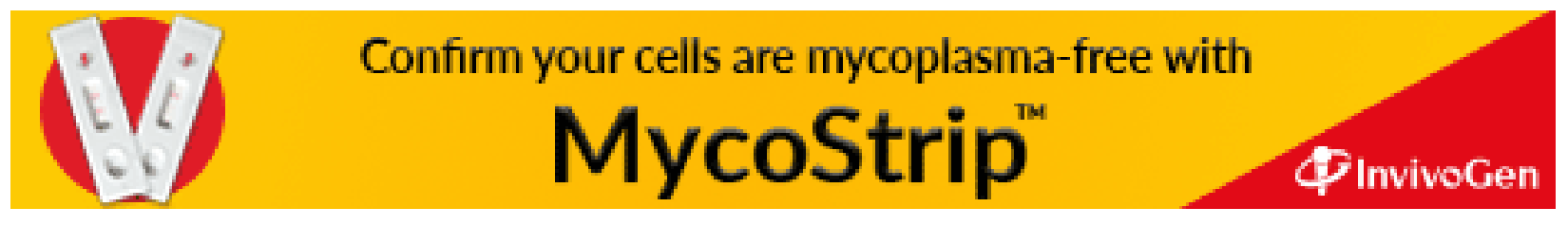

